Rev. Hist., $\mathrm{N}^{\circ} 27$, vol. 2, Julio-Diciembre 2020: 71-101

ISSN 0717-8832

https://doi.org/10.29393/RH27-12MIPC20012

\title{
Mortalidad infantil y sistema hospitalario en la Provincia de Santiago (1930-1973)
}

Infant mortality and hospital system in the Santiago Province (1930-1973)

Pablo Chávez Zúñiga: ORCID: http://orcid.org/0000 0002-1726-7954*

Víctor Brangier Peñailillo: http://orcid.org/0000-0003-3583-2221 ${ }^{* *}$

\section{RESUMEN}

Este artículo aborda las características del sistema hospitalario y sus vínculos con la mortalidad infantil en la Provincia de Santiago entre 1930 y 1973, con la finalidad de discutir los múltiples escenarios políticos y médicos, donde se intentó solucionar esta problemática de salud pública. La hipótesis de trabajo propone que la extensión de las instalaciones de salud y los progresos de varios parámetros clínicos contribuyeron a disminuir gradualmente la cantidad de muertes. La investigación utilizó fuentes de prensa, literatura especializada y decretos y proyectos de Ley del Ministerio de Salud. En las conclusiones se examina la circulación del conocimiento médico, la inserción de nuevas tecnologías y la profundización de las políticas preventivas, como elementos claves para mejorar la salud infantil.

Palabras clave: mortalidad infantil, hospitales de niños, sistema hospitalario, Santiago

\section{ABSTRACT}

This article addresses the characteristics of the hospital system and its links with infant mortality in the Province of Santiago between 1930 and 1973, in order to discuss the multiple political and medical scenarios, where attempted were made to solve this public health problem. The working hypothesis proposes that the expansion of health facilities and the progress of various clinical parameters contributed to gradually decrease the number of deaths. The research used press sources, specialized literature and decrees and bills of the Ministry of Health. The conclusions examine the circulation of medical knowledge, the insertion of new technologies and the deepening of preventive policies, as key elements to improve children's health.

Keywords: infant mortality, children's hospitals, hospital system, Santiago

Recibido: marzo 2020

Aceptado: octubre 2020

\footnotetext{
* Doctor en Historia, Universidad de Chile, Chile. Email: pablo.chavez.zuniga@gmail.com.

** Doctor en Historia, Universidad de Chile, Académico Universidad Bernardo O’Higgins, Chile. Email: vmbrangi@gmail.com.
} 


\section{Introducción}

Durante el siglo XX, la mortalidad infantil fue uno de los grandes problemas de salud pública. Chile registraba una de las tasas más altas de mortandad entre los países que elaboraban una estadística confiable. En la prensa, en las discusiones políticas y en los textos médicos aparecía como uno de los desafíos más problemáticos por resolver e intentar lograr una disminución de las cifras. Esta realidad se convierte en un novedoso objetivo historiográfico para tratar de comprender las transformaciones y las consecuencias históricas que generaron el proceso de medicalización a lo largo de cuatro décadas de la pasada centuria y estudiar los factores que demuestran la reducción en las cantidades de muertes infantiles. Desde esta plataforma surge el interés por abordar los efectos del sistema hospitalario en este profundo cambio histórico, la especialización de la medicina y sus relaciones con la cultura.

El estudio de esta temática se apoya en varias razones. Por un lado, este cuadro histórico desde arriba, destaca las explicaciones del fenómeno en Chile, lo que constituye un caso excepcional. Si se comparan los parámetros demográficos con los de algunas naciones, el país presentaba elevadas cifras de mortandad, altos índices de pobreza material, carencias en viviendas, falta de expansión de la red de alcantarillado, entre otras variables. Por otra parte, dada las proporciones de este escenario, los médicos y las autoridades políticas comenzaron a considerar la profundidad de esta problemática, aunque las respuestas fueron un poco lentas y sometidas a exámenes de ensayo y error. En cuanto a las características del sistema hospitalario durante este período, a medida que la medicina pediátrica fue aumentando el conocimiento de las enfermedades infantiles y, desde esa óptica científica, el Estado extendió las discusiones y soluciones sobre los problemas sociales-sanitarios que afectaban a la mayoría de la población en las ciudades.

Nuestro propósito con este artículo es abrir nuevas interrogantes para analizar las múltiples trayectorias de la mortalidad infantil en el país, al centrar las narrativas menos en la implementación de leyes o los discursos sobre la temática y más en los vínculos con el funcionamiento del sistema hospitalario, estudiado desde la ampliación de las redes asistenciales y el impacto, a lo largo de varias décadas, en las cifras de fallecimientos en la niñez. Al respecto, debe considerarse que la mayoría de las discusiones historiográficas se han preocupado en el devenir de varios escenarios que llevaron a la reducción de la mortandad, ya sea que profundicen en la fundación de hospitales infantiles o en las características de las viviendas a inicios del siglo XX. Sin lugar a dudas, estos abordajes aportan a la disciplina el relato de los factores que influyeron en las mejoras de la salud de los habitantes, pero existe un 
extenso campo hacia las variables clínicas y su anclaje a un contexto cultural que aún genera entradas susceptibles de tratar ${ }^{1}$.

Nuestra hipótesis de trabajo propone que la extensión de las instalaciones de salud y los progresos de varios parámetros clínicos contribuyeron a disminuir gradualmente la cantidad de muerte. De esa manera, interesa destacar que el aumento de la demanda por atenciones sanitarias a los infantes tuvo un profundo impacto sobre la estructura del sistema de salud, dejando a muchas personas carentes de recibir los cuidados médicos necesarios. Los aportes historiográficos de este período establecen la importancia de la mortandad en la niñez como una problemática de salud pública. En esa línea, los estudios acerca de la salud infantil, el desarrollo de los conocimientos pediátricos y los establecimientos hospitalarios, han tratado los orígenes de esta especialidad médica que dejó en evidencia la precaria condición en que se encontraban los lactantes ${ }^{2}$.

Desde el prisma de la historia cultural se interrogan las prácticas de cuidado en la infancia vinculadas a las atenciones familiares que rodearon a los recién nacidos, las que durante el siglo XIX se llevaron a cabo en el ámbito doméstico. En el siglo XX, estas mismas prácticas se efectuaron bajo la supervisión médica. En este sentido, se indaga el factor de la alimentación infantil, la nutrición en la niñez y los tratamientos médicos de las diarreas infantiles ${ }^{3}$. También se enfatiza el papel de la medicina como agente cultural. En ella los médicos se integran en el entramado social y adquieren un rol activo en su comunidad para mejorar la salud infantil ${ }^{4}$. De esta manera, los estudios de los procesos de salud y enfermedad ingresan a una temática que aporta acerca de las tramas socioculturales ${ }^{5}$.

Otra de las aristas sobre la mortalidad infantil se ha preocupado de estudiar el proceso de medicalización, a partir de dos dinámicas simultáneas: la circulación y aplicación del

\footnotetext{
${ }^{1}$ Rojas, Jorge. 2010. Historia de la infancia en el Chile republicano: 1810-2010, Santiago, Junta Nacional de Jardines Infantiles; Rojas, Jorge. 1999. "Trabajo infantil en la minería: apuntes históricos”, en Historia, vol. 32, Santiago, pp. 367-441; Rojas, Jorge. 1996. Los niños cristaleros: trabajo infantil de la industria. Chile, 1880-1950, Santiago, Dirección de Bibliotecas, Archivos y Museos; Rojas, Jorge. 2001. "Los niños y su historia: un acercamiento conceptual y teórico desde la historiografía", en Pensamiento Crítico. Revista electrónica de historia, № 1, Santiago, pp. 1-39.

2 Rodríguez, Esteban. 1998. "La construcción de la salud infantil. Ciencia, medicina y educación en la transición sanitaria en España", en Cuadernos de Historia Contemporánea, № 18, Madrid, pp. 19-52.

${ }^{3}$ Luna Freire, María Martha de. 2008. "Ser mãe é uma ciência': mulheres, médicos e a construção da maternidade científica na década de 1920", História, Ciências, Saúde-Manguinhos, vol. 15, Río de Janeiro, pp. 153-171; Luna Freire, María Martha de. 2010. "A construção da "maternidade científica" no Brasil republicano: Mulheres, mães e médicos: discurso maternalista no Brasil", Estudos Feministas, vol. 18/3, Florianópolis, pp. 941-964; Di Liscia, María Silvia. 2002. "Hijos sanos y legítimos: sobre matrimonio y asistencia social en Argentina (1935-1948)", História, Ciências, em Saúde-Manguinhos, vol. 9, Río de Janeiro, pp. 209-232.

${ }^{4}$ Rodríguez, Esteban. 2003. "La salud infantil, asunto ejemplar en la historiografía contemporánea", en Revista Dynamis, № 23, Barcelona, pp. 27-36.

${ }^{5}$ Armus, Diego. 2007. La ciudad impura. Salud, tuberculosis y cultura en Buenos Aires, 1870-1950, Buenos Aires, Edhasa.
} 
conocimiento europeo, por un lado, y el espacio local de la población trabajada, por el otro ${ }^{6}$. Desde esta base, se realiza un seguimiento a los debates, el desarrollo de políticas públicas de salud efectuadas por los pediatras ${ }^{7}$. Asimismo, al distinguir el porcentaje de mortalidad infantil, en comparación con los fallecimientos generales, lleva a establecer que en la década de 1930 comenzó un descenso sostenido de las muertes tras el nacimiento debido al empleo de varias técnicas que permitieron el control de la mortandad durante el parto y la posnatal ${ }^{8}$. Dichos análisis se han centrado en las soluciones que el aparataje estatal dirigió para superar las problemáticas de la infancia y mejorar el ambiente en que se encontraban las familias más pobres, lo que se expresó en el surgimiento de los servicios sociales y la extensión de instalaciones como el alcantarillado, la vivienda y el acceso al agua potable?

Las interrogantes sobre la mortalidad infantil, en el caso de Chile, se han abordado desde la cultura y rescatando que las agresiones físicas, la mala nutrición y el abandono eran variables extendidas hasta mediados del siglo $X X X^{10}$. A ello se agregaban las altas tasas de mortandad en el parto, la baja difusión de consejos médicos de crianza y la carencia de establecimientos hospitalarios para el cuidado de la niñez ${ }^{11}$. Lo anterior se reforzaba por un desconocimiento generalizado de la higiene infantil, lo que repercutía en el surgimiento de enfermedades en el

${ }^{6}$ Gallo, Óscar y Márquez, Jorge. 2011. "La mortalidad infantil y la medicalización de la infancia. El caso de Titiribí, Antioquía, 1910-1950”, en Revista Historia y Sociedad, № 20, Medellín, pp. 57-89.

7 Birn, Anne-Emanuelle. 2008. "Doctors on record: Uruguay's infant mortality stagnation and its remedies, 18951945", in Bulletin of the History of Medicine, № 82, Maryland, pp. 311-354; Birn, Anne-Emanuelle. 2007. "Child health in Latin America: historiographic perspectives and challenges", em História, Ciências, Saúde-Manguinhos, vol. 14/3, Río de Janeiro, pp. 677-708; Birn, Anne-Emanuelle, Pollero, Raquel y Cabella, Wanda. 2003. "No se debe llorar sobre la leche derramada: el pensamiento epidemiológico y la mortalidad infantil en Uruguay, 1900-1940", en Estudios interdisciplinarios de América Latina y el Caribe, vol. 14/1, Tel Aviv, pp. 35-65.

8 Carbonetti, Adrián y Peranovich, Andrés. 2007. "La mortalidad infantil en la ciudad de Córdoba entre principios y mediados del siglo XX", en Carbonetti, Adrián (compilador), Historias de enfermedad en Córdoba desde la colonia hasta el siglo XX, Córdoba, Programa de Salud y Enfermedad, Centro de Estudios Avanzados, U.N.C., pp. 75-93.

${ }^{9}$ Netto, Eduardo. 2012. "The lives of children and their public intimacy: social work as a new factor in attending to the needs of children in Latin America, 1928-1948", em História, Ciências, Saúde-Manguinhos, vol. 19/2, Río de Janeiro, pp. 1-23; Ehrick, Christine. 2001. "Mothers and the Colossal Machine: Feminism, Social Assistance and the State in Uruguay, 1910-1932", in The Americas, vol. 58/1, pp. 121-139; Kiple, Kenneth. 1989. "The Nutritional Link with Slave Infant and Child Mortality in Brazil", in The Hispanic American Historical Review, vol. 69/4, pp. 677-690.

10 Osta, María Laura. 2016. "Niños y niñas, expósitos y huérfanos en Montevideo del siglo XIX", en Revista de la Facultad de Derecho, № 41, Montevideo, pp. 155-189; Milanich, Nara. 2011. "Women, children, and domestic labor in nineteenth-century Chile", in Hispanic American Historical Review, vol. 91/1, Durham, pp. 29-62; Palma, Patricia. 2009. "Mortalidad infantil en Chile. La situación médica social en la Casa de Expósitos de Santiago 1889-1899", en Anales de historia de la medicina, vol. 19/2, Santiago, pp. 127-137; Milanich, Nara. 2009. Children of fate. Childhood, class, and the State in Chile, 1850-1930, Durham, Duke University Press.

11 Zárate, Soledad. 2010. "El licor de la vida. Lactancia y alimentación materno-infantil en Chile, 1900-1950", en Sciolla, Carolina (compiladora), Historia y cultura de la alimentación en Chile, Santiago, Catalonia; Zárate, Soledad. 2005. "Parto, crianza y pobreza en Chile", en Sagredo, Rafael y Gazmuri, Cristián (directores) Historia de la vida privada en Chile. Tomo III: el Chile contemporáneo de 1925 a nuestros días, Santiago, Aguilar Chilena Ediciones Taurus. 
primer año de vida ${ }^{12}$. Estas circunstancias, desde el punto de vista de la historia social, permiten ingresar a la situación material paupérrima en que habitaban los infantes ${ }^{13}$. Sumado a ello, una de las problemáticas se relaciona con los niños trabajadores. El conflicto se producía por la precariedad y peligrosidad de las faenas industriales, donde los pequeños que laboraban se encontraban en alto riesgo de accidentes.

La documentación estatal, los periódicos y las publicaciones especializadas permiten trazar una trayectoria probable de la mortalidad en la infancia y los vínculos con la infraestructura hospitalaria. Con esa finalidad, el análisis considera registros históricos provenientes de la prensa escrita por la amplia cantidad de contenidos acerca de la mortandad en la niñez, la ampliación de la atención médica infantil y los hitos relevantes que condujeron a una reducción de los fallecimientos. También contempla bibliografía disciplinaria, lo que permite ingresar, desde la perspectiva científica, a las descripciones del estado sanitario de los infantes y entregan características de esta problemática de salud pública en la niñez. Además, toma en cuenta documentos del Ministerio de Salud, los que comprenden decretos y normas sobre el funcionamiento de los hospitales. Este material se conserva en el Archivo Nacional de la Administración, específicamente en el Fondo de Ministerios y Servicios Públicos.

Este artículo se organiza en cuatro secciones: en la primera abordamos el desarrollo de las políticas de salud en la niñez, la implementación de organismos estatales y el quiebre que se produjo desde el punto de vista institucional con la creación del Servicio Nacional de Salud; en la segunda examinamos algunos indicadores de la salud infantil como las causales de mortandad, las condiciones precarias en que se encontraba el sistema hospitalario y las dificultades para difundir los preceptos higiénicos durante la crianza infantil; en la tercera estudiamos las complicaciones que existían en el funcionamiento de los hospitales infantiles, el colapso de los recintos y la extensión de las postas en el radio urbano; por último, tratamos la distribución de especialistas médicos y el arribo de nuevas tecnologías hospitalarias lo que permitió frenar, en parte, la alta mortalidad infantil.

\footnotetext{
12 Cavieres, Eduardo. 2001. "Ser infante en el pasado. Triunfo de la vida o persistencia de estructuras sociales. La mortalidad infantil en Valparaíso, 1880-1950", en Revista de historia social y de las mentalidades, № 5, Santiago, pp. 31-58; Cavieres, Eduardo. 1999. "Salud Pública. Estrategias, políticas fiscales y cambio cultural en la reducción de la mortalidad. Valparaíso, 1920-1960”, en Cuadernos de Historia № 19, Santiago, pp. 195-224.

${ }^{13}$ Benedetti, Laura. 2019. "El hambre, la miseria y la escuela: el surgimiento de las organizaciones de auxilio a la infancia escolar pobre de Concepción (1917-1929)", en Revista Historia Universidad de Concepción, № 26/2, Concepción, pp. 33-52; Salazar, Gabriel. 2007. Ser niño "huacho" en la historia de Chile (siglo XIX), Santiago, LOM Ediciones; Salazar, Gabriel y Pinto, Julio. 2002. Historia contemporánea de Chile V. Niñez y juventud, construcción cultural de actores emergentes, Santiago, LOM Ediciones; Illanes, María Angélica. 1999. "Maternalismo popular e hibridación cultural en Chile 1900-1920", en Nomadías, № 1, Santiago, pp.185-211; Illanes, María Angélica. 1993. En el nombre del pueblo, del estado y de la ciencia: historia social de la salud pública, Chile, 1880-1973: hacia una historia social del Siglo XX, Santiago, Colectivo de Atención Primaria.
} 


\section{La administración política en el ámbito de la salud infantil}

La participación del Estado en la Beneficencia provenía desde la época colonial. El gobierno de Manuel Bulnes dispuso la creación de las Juntas Directoras de Hospitales, denominadas de Beneficencia a partir de 1844, la que intervenía en la creación y marcha de los establecimientos. Así, a lo largo del siglo XIX, los recursos y gastos que originaban los recintos benéficos eran financiados en parte por la caridad privada, siendo aportes que en algunos años fueron más o menos equivalentes a las cuotas fiscales ${ }^{14}$. Al inicio de la nueva centuria, junto con la fundación de hospitales infantiles, se produjo un cambio en la administración de los hospitales y en el concepto de paciente, desde el ingreso solo para esperar el deceso hacia espacios en los que era posible encontrar mayores competencias médicas, mejores técnicas para el diagnóstico y el tratamiento científico ${ }^{15}$. Sin embargo, según el médico Alejandro del Río, cada año la Junta presentaba considerables déficits económicos para responder a las demandas hospitalarias, las "que han tenido que ver seriamente resentidos sus servicios de recepción de enfermos, debido a que no cuentan con los medios más indispensables para su atención" ${ }^{16}$.

En 1938, con la promulgación de la Ley de medicina preventiva quedó reflejada la consolidación del Estado en materia de salubridad e higiene pública y el retroceso de la esfera benéfica en esta área. Esta legislación tendía a modificar y complementar el enfoque de medicina curativa practicada en los hospitales, donde el eje de la prevención se basaba en evitar la aparición de una dolencia o curarla en sus fases tempranas, antes de que se convierta en un mal peligroso. Los tres pilares de esta propuesta eran: el examen médico de carácter periódico, la extensión de la educación sanitaria y la profilaxia en los tratamientos ${ }^{17}$. En efecto, el objetivo de este proyecto era disminuir la alta morbilidad por la dificultad en evitar las enfermedades, "que encuentran su mejor medio de incubación y crecimiento en las condiciones de vida y materiales de una gran parte de la población. Salta a la vista la necesidad de instaurar una política de prevención que ataque en sus causas y cure en su comienzo las enfermedades" ${ }^{18}$.

A fines de la década de 1930, la Caja de Seguro Obrero era una de las instituciones de previsión social más importante del país. Entre sus múltiples funciones, el Servicio de Asistencia

\footnotetext{
${ }^{14}$ Ponce de León, Macarena. 2007. La Reforma de la Caridad Ilustrada: del Socorro Intramuros al Socorro Extramuros. Prácticas de Caridad en Santiago, 1830-1880, Pontificia Universidad Católica de Chile, Tesis para optar al Grado de Doctor en Historia, p. 9.

15 Chávez, Pablo y Brangier, Víctor. 2020. "Esa diaria gotera de la muerte: Mortalidad infantil y los albores de la medicina pediátrica en la provincia de Santiago, Chile, 1870-1913", en Salud Colectiva, vol. 16, Buenos Aires, p. 7.

${ }^{16}$ BN, La Nación. Santiago, 20 de octubre de 1933.

${ }^{17}$ Comunicación del doctor Federico Patiño Mac Iver, Jefe del Departamento de Bienestar de la Madre y del Niño dirigida a la Dirección General de Sanidad, informa sobre la mortalidad infantil y presenta proyecto de defensa del niño. Santiago, 27 de diciembre de 1935. ARNAD, vol. 110, s/f.

${ }^{18}$ BN, El Diario llustrado. Santiago, 23 de julio de 1937.
} 
de la Madre y del Niño, tenía por finalidad desarrollar puericultura pre y postnatal ${ }^{19}$. El aumento de los aportes estatales permitió que la Caja entregara atención médica a más de cincuenta mil infantes debido a la extensión de los servicios, "esta se prestará a la mujer embarazada y a los niños, hasta los dos años, o sea, justamente en el período en que es mayor la mortalidad infantil”20. Según el médico pediatra Honorio Aguirre, la mortalidad en la infancia presentaba cierta disminución por la acción de la Caja, "al controlar alrededor de 70.000 niños menores de 2 años en todo el país"21.

En cuanto a los organismos estatales creados para frenar la mortandad en la infancia, el Departamento de Asistencia Social de la Infancia, inaugurado en 1938, tuvo por objetivo ocuparse de la salud de la madre y el niño hasta la edad escolar ${ }^{22}$, fundando una red de "clínicas pre natales, maternidades, creches, jardines infantiles, hogares, controlando la distribución de la leche y protegiendo a la madre y al niño" ${ }^{23}$. Junto con ello, el organismo se ocupó por asegurar el cumplimiento de las leyes sociales que se relacionaban con la protección de ambos actores ${ }^{24}$. Esta política impulsada por el ministro de Salubridad, Eduardo Cruz-Coke, dispuso la instalación de Gotas de Leche, Consultorios del Seguro Obrero y Centros Preventivos de Sanidad en diversos barrios de la capital, evitando la duplicación de instituciones con fines similares o la existencia de sectores desprovistos de estos espacios de apoyo social. También, el Ministerio de Asistencia Social estaba preocupado por la protección de la maternidad, de la infancia y la adolescencia ${ }^{25}$. Toda esta organización era de vital importancia para implementar los preceptos de la medicina preventiva y difundir los cuidados infantiles entre amplios segmentos de la población, principalmente a través de los llamados Centros Materno-Infantiles en que se proporcionaba "atención médica a las embarazadas durante todo el período de gestación y posteriormente se controla al niño en la edad de la lactancia y en la época pre escolar" ${ }^{26}$.

\footnotetext{
${ }^{19}$ Bustamante, Werner. 1939. Contribución al estudio del recién nacido y del lactante chileno (Santiago), Universidad de Chile, Tesis para optar al título de médico cirujano, p. 18.

${ }^{20}$ BN, La Nación. Santiago, 30 de mayo de 1937.

${ }^{21}$ BN, La Nación. Santiago, 7 de diciembre de 1945.

22 El Servicio Dental Escolar Obligatorio fue una de las instituciones encargadas de la protección de la infancia. Para seguir las funciones de este organismo, la cantidad de niños atendidos y los efectos de su implementación, véase Mensaje del Ministerio de Salud Pública sobre la labor del Servicio Dental Escolar Obligatorio. Santiago, 13 de agosto de 1935. Archivo Nacional de la Administración, Fondos de Ministerios y Servicios Públicos, Ministerio de Salud, vol. $17, \mathrm{~s} / \mathrm{f}$ (en adelante ARNAD).

${ }^{23}$ BN, El Mercurio. Santiago, 26 de mayo de 1938.

${ }^{24}$ Charla del doctor Alfaro sobre la labor de los centros preventivos del Departamento de Bienestar de la Madre y del Niño de la Dirección General de Sanidad. Santiago, 13 de septiembre de 1939. ARNAD, vol. 225, s/f.

${ }^{25}$ ARNAD Decreto que crea la Dirección General de Protección a la Infancia y Adolescencia. Santiago, 7 de octubre de 1942., vol. 387, s/f.

${ }^{26}$ ARNAD Actividades de la Dirección General de Protección a la Infancia y Adolescencia. Santiago, 18 de marzo de 1952., vol. 1 195, s/f.
} 
Años más tarde, el Departamento Central de Protección a la Madre y al Niño impulsó planes para modernizar y hacer más eficientes las funciones a su cargo ${ }^{27}$. No obstante, en la práctica los órganos no tenían las atribuciones en el control de los servicios ni para unificar los programas de trabajo o coordinar sus acciones. Ante eso, se llevaron a cabo reformas propuestas por el ministro de Salubridad, doctor Salvador Allende, declarando que el cuidado a la madre y el niño estaban obstruidas por "la disparidad de criterios en lo que se refiere a métodos preventivos de inmunización o alimentación, perjudica la obra de conjunto, produce estadísticas diferentes que desorientan al Ministerio del ramo" ${ }^{28}$.

El trabajo de los poderes públicos, desde inicios del siglo XX, se tradujo en la formación de múltiples organismos de asistencia al niño ${ }^{29}$, tales como el Seguro Obrero, la Beneficencia Pública, el Patronato Nacional de la Infancia, el Consejo de Defensa del Niño, la Dirección de Protección a la Infancia y Adolescencia, la Cruz Roja ${ }^{30}$. A pesar de los intentos por organizarlos, todos ellos funcionaban de manera simultánea y generaban ingentes gastos de dineros fiscales, además rebasadas por la precaria situación económica en que se hallaban los habitantes. La estructura sanitaria era considerada "inconexa, no está conformada a comando único, no obedece a un plan general, y tampoco dispone de recursos materiales que guarden relación con la obra que debe ejecutarse para salvaguardar la salud"31.

Según el médico Arturo Baeza Goñi, "falta todavía un largo camino que recorrer para llegar a un estado más o menos satisfactorio de higiene pública y social. Para demostrar esta aseveración basta revisar la condición en que ingresan los niños que acuden a los hospitales, donde la mayoría son enfermos por alimentación insuficiente y basta analizar los antecedentes para comprobar que toman una escasa cantidad de leche, que los mayores no conocen ni la mantequilla ni la carne ${ }^{\prime 32}$. Una de las causales de la desnutrición en los niños, a juicio del mismo facultativo, se encontraba en "la ignorancia de los padres que no saben cuidarlos ni alimentarlos en forma adecuada, esto los deja indefensos al ataque de las infecciones del ambiente malsano"33. Así, se añadía la insuficiencia de las redes de alcantarillado o acceso al agua potable, lo que debido al acelerado crecimiento de la planta urbana y los habitantes, dejaba fuera de estos servicios a extensos segmentos de la comunidad.

\footnotetext{
${ }^{27}$ El problema de la atención maternal. Santiago, 1 de febrero de 1943. ARNAD, vol. 470, s/f.

${ }^{28}$ BN, La Nación. Santiago, 16 de abril de 1940.

29 Llanos, Claudio y Lanfranco, María Fernanda. 2017. "La discusión política sobre la mortalidad infantil en Chile durante la década de 1930. Elementos para una aproximación histórico política", en Anuario de Estudios Americanos, vol. 74/2, Sevilla, p. 686.

${ }^{30}$ ARNAD, Presentación Proyecto de Ley para creación de Junta Central de Protección a la Infancia. Santiago, 27 de diciembre de 1937. vol. 17, s/f.

${ }^{31}$ BN, La Nación. Santiago, 18 de enero de 1936.

${ }^{32}$ BN, El Mercurio. Santiago, 12 de marzo de 1945.

${ }^{33}$ BN, El Mercurio. Santiago, 9 de noviembre de 1962.
} 
En 1952, la Ley № 10.383 creó el Servicio Nacional de Salud (en adelante SNS), lo que marcó un cambio trascendental a las deficientes condiciones sanitarias de las ciudades. El texto legal comprendía tres variables fundamentales: primero, fortalecer la protección de la salud, es decir, fomentar acciones preventivas mediante el saneamiento ambiental, extender la red de alcantarillado y profundizar el acceso de agua potable a viviendas que carecían de condiciones de salubridad mínimas; segundo, reforzar el ámbito de la salud, sobre todo el problema que significaba la higiene materno infantil con la finalidad de reducir la mortalidad y la morbilidad infantil, lo que se logró en las décadas siguientes con la disminución de la desnutrición y mejoras en el entorno de los infantes ${ }^{34}$; tercero, funciones de recuperación de la salud, a través de la atención de las enfermedades una vez ocurridas, en los consultorios, los hospitales o en el domicilio de los afectados.

Para el médico René Valle, Director del Hospital Luis Calvo Mackenna, la implementación del SNS requería de individuos técnicos debido a que "se carece de enfermeras hospitalarias porque se dispone de pocas escuelas, se adolece del personal idóneo de laboratorios y auxiliares de enfermería. Se han dado ya los primeros impulsos para la creación de escuelas que provean de este personal especializado" ${ }^{35}$. Además, el SNS generó la fusión de varios servicios encargados de la protección de la niñez, otorgando mayores aportes fiscales, igualando criterios, ajustando la distribución de instituciones, suprimiendo duplicidades costosas para el fisco y ejerciendo un control administrativo que modernizó la entrega de salud a los habitantes $^{36}$. Una política de defensa materno-infantil debía cumplir tres ejes fundamentales: primero, trabajar de manera unitaria, en que las acciones fueran dirigidas por la esfera estatal a través de procedimientos estandarizados; segundo, fomentar un sentido integral donde cada una de las etapas de la infancia sea uniforme y constantemente atendida para evitar que el lactante, pre escolar o escolar sea abandonado ${ }^{37}$; tercero, mantener un carácter intensivo en que el Estado movilice sus recursos para la ejecución de proyectos.

\section{Indicadores demográficos y sanitarios de la infancia}

En la constitución de la mortalidad infantil como un problema de salud pública, el desconocimiento de los síntomas de las enfermedades fue un agente que contribuyó a la separación entre los saberes médicos y los habitantes. En muchos casos, "los niños mueren porque su mal no ha sido reconocido a tiempo, no ha sido indicado rápidamente al médico o

\footnotetext{
${ }^{34}$ ARNAD, Entrega de facultades al Ministro de Salud para que suscriba convenios en representación del gobierno de Chile con la Corporación para la nutrición infantil. Santiago, 3 de noviembre de 1976. vol. 1 922, s/f.

35 BN, El Mercurio. Santiago, 28 de febrero de 1953.

${ }^{36}$ Figueroa, Ernesto. 1951. El consultorio externo del Hospital de niños y su misión médico-social, Santiago, [s.n.], p. 7.

${ }^{37}$ ARNAD, Aprobación de Reglamento del Servicio Médico Escolar, de la Dirección General de Protección a la Infancia y Adolescencia. Santiago, 5 de noviembre de 1943. vol. 455, s/f.
} 
desde el principio no sido cuidado debidamente" ${ }^{\prime 38}$. La construcción de registros sanitarios resultó bastante complejo, sobre todo para definir los criterios que permitieran clasificar las enfermedades infantiles ${ }^{39}$. Con esas evidencias cuantitativas, la disciplina pediátrica logró obtener más conocimientos sobre las afecciones infantiles y sus tratamientos. Sin duda, estos cuadros tuvieron impactos diferentes dependiendo de la edad del recién nacido. En la mortalidad de los menores de un mes jugaba un rol importante las causas denominadas congénitas o maternas, es decir, factores derivados del embarazo y principalmente del parto ${ }^{40}$; en cambio, los fallecimientos entre un mes y el año se veían influidos por elementos ambientales como las infecciones o la alimentación ${ }^{41}$.

Los números de la tabla 1 reproducen las principales causales de muertes infantiles entregadas por el Anuario Estadístico. La cuantificación médica recogió las causales anuales, aunque los conceptos tuvieron variaciones a lo largo de los años, lo que explica la falta de continuidad de las cifras en este período. Según esta tabla, hasta mediados de la década de 1950 una de las principales categorías anotadas correspondió al nacimiento prematuro o los efectos de las complicaciones del parto. Desde 1956 esta definición fue ingresada a otras enfermedades de la primera infancia. Además, en esta década se produjo un mejoramiento en las técnicas de cuantificación, lo que aumentó la capacidad de recolección estadística y lógicamente, los números de diagnósticos precisos sobre las muertes también crecieron. Así, entre 1956 a 1973, los principales males que llevaron a la muerte en la niñez fueron las de tipos respiratorios, gastrointestinales y afecciones particulares en los recién nacidos.

Durante la primera mitad del siglo XX, la anotación de las causales de mortandad experimentó cambios importantes. Con el tiempo, la ausencia de declaración médica en las circunstancias de muerte se transformó en un obstáculo para desarrollar una estadística confiable. Por ejemplo, en 1959 "las defunciones de menores de un año, solamente tenía certificación médica de la causa de fallecimiento el 51.4 \%. Esto es equivalente a decir que no sabemos nada concreto sobre los determinantes de la muerte en la mitad de los niños de esta edad"42. Esta inexistencia y en algunos casos errores en los diagnósticos clínicos era un

\footnotetext{
${ }^{38} \mathrm{BN}$, El Mercurio. Santiago, 15 de mayo de 1930.

${ }^{39}$ Chávez, Pablo y Soto, José. 2019. "La primera estadística sanitaria infantil de la provincia de Santiago de Chile (1860-1929)", en Dynamis, vol. 39/2, Barcelona, p. 153; Bernabeu, José, Fariñas, Diego, Sanz, Alberto y Robles, Elena. 2003. "El análisis histórico de la mortalidad por causas. Problemas y soluciones", en Revista de Demografía Histórica, vol. XXI/1, Barcelona, pp. 167-193.

${ }^{40}$ Meneghello, Julio, Rosselot, Jorge y Manterola, Alejandro. 1961. Lecciones de pediatría clínica y social. Puericultura individual y colectiva, Santiago, Editorial Universitaria, p. 10; Meneghello, Julio. 1960. Lecciones de pediatría. Tomo II: patología y clínica del prematuro y lactante, Santiago, Editorial Universitaria.

${ }^{41}$ Lagos, Rubén. 1952. El control prenatal y el recién nacido, Santiago, Tesis para optar al grado de Licenciado en Medicina, p. 15.

42 Behm Rosas, Hugo. 1962. Mortalidad infantil y nivel de vida, Santiago, Ediciones de la Universidad de Chile, p. 62.
} 
problema porque la mortandad en este segmento de edad representaba la tercera parte del total de las defunciones.

Tabla 1. Causales de muertes infantiles en Provincia de Santiago, 1931 - 1973

\begin{tabular}{|c|c|c|c|c|c|c|c|c|c|c|c|c|c|}
\hline Causal de & & & & & & & Años & & & & & & \\
\hline & 1931 & 1934 & 1938 & 1942 & 1947 & 1951 & 1956 & 1958 & 1963 & 1965 & 1969 & 1971 & 1973 \\
\hline $\begin{array}{l}\text { Nacimiento } \\
\text { prematuro o } \\
\text { consecuencias } \\
\text { del parto }\end{array}$ & 496 & 473 & 719 & 562 & 656 & 544 & 341 & - & - & - & - & - & - \\
\hline $\begin{array}{l}\text { Afelectasia y } \\
\text { asfixia durante o } \\
\text { después del } \\
\text { nacimiento }\end{array}$ & - & - & - & 200 & 141 & - & - & - & - & - & - & - & - \\
\hline Neumonía & - & - & - & - & - & - & 1728 & 1961 & 1534 & 1292 & 8026 & 5308 & 4184 \\
\hline $\begin{array}{l}\text { Gastritis, } \\
\text { enteritis y colitis, } \\
\text { salvo diarreas } \\
\text { del recién nacido }\end{array}$ & - & - & - & - & - & - & 295 & & 1450 & 1252 & 3499 & 3056 & 2192 \\
\hline $\begin{array}{l}\text { Otras } \\
\text { enfermedades } \\
\text { particulares de la } \\
\text { primera infancia }\end{array}$ & - & - & - & - & - & - & 2023 & 2997 & 1220 & 1199 & 1800 & 1920 & 2911 \\
\hline
\end{tabular}

Fuente: Elaboración de los autores a partir del Anuario Estadístico de la República de Chile, 1930-1973.

Sin lugar a dudas, la capital y las ciudades de Chile adolecían de graves insuficiencias en los servicios hospitalarios en general. La situación en los recintos era dramática, "no hay gasa ni yodo ni algodón en muchos de ellos y los médicos deben acudir al pabellón de operaciones con sus propios instrumentos. En otros hospitales, las medicinas que recetan los médicos han de ser enviadas a comprar a las farmacias privadas, pues los botiquines hospitalarios están vacíos" ${ }^{\prime 3}$. Así, la atención de los enfermos se efectuaba de modo precario y los elementos de que se disponían no guardaban relación numérica con la extensión y la proporción de los habitantes infantiles a los que estaban destinados. Entre los nacimientos era frecuente la falta de asistencia primaria durante la preñez, en el parto y en los primeros meses de vida del niño.

En esta época, las matronas desempeñaban su oficio en hogares donde no hallaban ningún material sanitario para ejercer su labor. Una visitadora de enfermos afirmaba que "he visto

${ }^{43}$ BN, El Mercurio. Santiago, 5 de noviembre de 1955. 
llegar al mundo a miles de criaturas sanas y ser recibidas en papeles de diarios; más tarde cuando he vuelto a los días a comprobar cómo fue atendida la enferma, la he encontrado casi siempre sana en sus quehaceres domésticos y la criatura pálida, ya víctima del ambiente sucio, sin bañarla y esa vivienda inmunda ha empezado su obra mortal" 44 .

A lo anterior, se agregaba que a inicios de la década de 1940, "un $60 \%$ de las parturientas no tiene servicio de maternidad al cual acudir y debe pasar el parto en el propio hogar, casi siempre carente de condiciones adecuadas para ese trance" ${ }^{45}$. El resultado fue que las madres corrían grandes riesgos vitales y sus hijos recién nacidos no obtenían una oportuna atención obstétrica ${ }^{46}$. En un artículo titulado "El aspecto médico social del Problema del Niño", el médico Guillermo Morales Beltrami entregaba estadísticas de esta situación, en cifras "la población de lactantes, puede calcularse en unos 200 mil niños, de los cuales 60 mil son atendidos en buena forma en su casa y 50 mil por las instituciones públicas, semifiscales y particulares de protección a la infancia. La conclusión es dolorosa; en Chile existen 90 mil niños que no reciben atención alguna hasta los dos años de edad, hecho que explica el enorme número de víctimas que en este período incuban" 47 .

Frente a ese contexto, era fundamental implementar maternidades con todos los elementos necesarios para una intervención positiva. Los médicos diagnosticaron "un grave déficit de camas con los consiguientes reclamos que obligan a las madres a tener un parto en condiciones precarias, en manos de personas no profesionales y en ambientes propicios a toda clase de infecciones" ${ }^{\prime 8}$. Hasta ese momento, la situación material de las maternidades era considerada como grave, en primer lugar, el personal especializado era escaso, ya fueran obstetras, pediatras, matronas, auxiliares $y$, en segundo lugar, las deficiencias de recursos como pabellones quirúrgicos, salas de recuperación, servicios de puericultura y de prematuros, incubadoras, aparatos de resucitación, o sea aparatos básicos para salvar vidas.

En la capital se presentaban muchos enfermos cuyo estado de salud exigía de manera urgente el ingreso hospitalario, no obstante "por falta de camas y servicios en los establecimientos de Beneficencia, se ven forzados a permanecer en sus hogares, con perjuicio gravísimo para su salud, ya que las personas no están en condiciones de recibir atención médica, ni de medicinarse o seguir los tratamientos ordenados por los facultativos en sus propios hogares $^{\prime 49}$. Esta situación dejaba a gran cantidad de enfermos sin obtener los estados

\footnotetext{
${ }^{44}$ BN, El Diario llustrado. Santiago, 4 de septiembre de 1937.

45 BN, El Mercurio. Santiago, 6 de junio de 1941.

${ }^{46}$ Zárate, Soledad. 2008. Dar a luz en Chile, siglo XIX. De la "ciencia de la hembra" a la ciencia obstétrica, Santiago, Ediciones Universidad Alberto Hurtado; Zárate, Soledad. 2007. "De partera a matrona. Hacia la asistencia profesional del parto en Chile en el siglo XIX", en Calidad en la educación, № 27, Santiago, pp. 284-297.

${ }^{47}$ BN, La Nación. Santiago, 11 de diciembre de 1940.

${ }^{48}$ BN, El Mercurio. Santiago, 6 de agosto de 1963.

${ }^{49}$ BN, La Opinión. Santiago, 11 de noviembre de 1943.
} 
elementales de higienización, reposo o aislamiento médico necesario. Esto último se complicaba cuando los niños adquirían enfermedades contagiosas y al transformarse el hogar en un foco de peligro, tanto por su capacidad de contagiar o ser contagiados ${ }^{50}$. De esa manera, se exhibía un "extenso sector de la población infantil sin acceso a las atenciones de salud que aparece expuesto a un elevado riesgo de morir en el primer año de vida" ${ }^{51}$.

Uno de los problemas relacionado con el cuidado médico era la falta de camas en número suficiente en los hospitales. Pese a las planificaciones en el ámbito de la salud, siguió habiendo un amplio sector de la población que no recibía cuidados médicos. En 1900, el país tenía unas 2.200 camas de adultos y virtualmente ninguna para individuos menores. En la Casa de Huérfanos, que reemplazaba una parte mínima de las necesidades, un elevado porcentaje de criaturas fallecían en el establecimiento ${ }^{52}$. En más de una ocasión se produjo "que una madre haya dado a luz en la puerta del hospital en que se le acaba de rechazar por imposibilidad de darle cabida" ${ }^{53}$. A inicios de la década de 1960, "más de dos tercios de los nacimientos está ocurriendo en instituciones públicas, y esta cantidad va acrecentándose. A pesar de ello, varias maternidades se ven obligadas a albergar dos mujeres por cama" ${ }^{54}$. Situación que años más tarde fue confirmada por el Ministro de Salud, Ramón Valdivieso indicando que "en algunas maternidades hay dos madres por cama, lo cual es un espectáculo denigrante" ${ }^{55}$.

La disposición de camas hospitalarias no solo fue presionada por el crecimiento de la población, sino que durante la estación estival recrudecían las afecciones gastrointestinales, lo que aumentaba la afluencia y gravedad de los enfermos respecto a otras épocas del año ${ }^{56}$. Esto último se tradujo en "una prolongación de la estadía hospitalaria y la consiguiente menor disponibilidad de camas, y como natural derivación un elevado coeficiente de mortalidad" ${ }^{57}$. A tal extremo llegaba la falta de recursos, que el SNS estuvo obligado "a contratar camas en clínicas particulares para atender los angustiosos pedidos de los enfermos, sobre todo a las parturientas. Por esta razón solo se deja en los recintos hospitalarios a los enfermos más graves, el resto debe convalecer en sus hogares que generalmente no reúnen las condiciones sanitarias requeridas" 58 .

\footnotetext{
${ }^{50}$ Scroggie, Arturo. 1958. Tratado de pediatría: enfermedades infecciosas, Santiago, Editorial Andrés Bello, Editorial Universitaria, p. 7.

${ }^{51}$ Behm Rosas, Hugo. 1962. Mortalidad infantil y nivel de vida, p. 80.

${ }^{52}$ Chávez, Pablo y Soto, José. 2019. "Historia de la mortalidad infantil en la Casa de Huérfanos de Santiago (18981923), en Anuario de Estudios Americanos, vol. 76/2, Sevilla, pp. 676-677.

53 BN, El Siglo. Santiago, 28 de diciembre de 1947.

${ }^{54}$ BN, El Mercurio. Santiago, 24 de agosto de 1960.

55 BN, El Mercurio. Santiago, 5 de diciembre de 1964.

56 Chávez, Pablo. 2020. "La mortalidad infantil: entre la alimentación y las enfermedades gastrointestinales en Santiago (1880-1920)", en Cuadernos de Historia, № 52, Santiago, pp. 69-101.

${ }^{57}$ BN, El Mercurio. Santiago, 10 de enero de 1962.

${ }^{58}$ BN, El Clarín. Santiago, 17 de noviembre de 1967.
} 
La presión sobre el sistema hospitalario obligó a los establecimientos a destinar camas y equipos de especialistas hacia enfermedades que tenían carácter estacional. Un pediatra del hospital Roberto del Río indicaba que "se han cerrado salas, porque el personal se ha ido movilizando hacia la lucha contra la influenza. Nuestro hospital atiende 200 mil casos por año con ocho mil hospitalizaciones en 400 camas, es como un acordeón. Si hay mucha diarrea, aumentan las salas de diarrea; si hay mucha influenza, se aumentan las salas de influenza" ${ }^{29}$. Este tipo modificó el antiguo criterio de administración hospitalaria en que se destinaba una sala para una determinada enfermedad y allí se admitían solo pacientes de una afección durante todo el año. La disposición dinámica de camas, según la estacionalidad, permitió hacer frente al aumento de enfermedades gastrointestinales en verano y a las de carácter respiratorio en invierno ${ }^{60}$.

En la década de 1960, el SNS elaboró una estrategia que intentaría reducir la mortalidad infantil, principalmente la ocurrida por diarreas ${ }^{61}$. Según este organismo "en 1966, fallecieron entre 12 y 15 mil niños debido a las diarreas infantiles y sus secuelas (deshidratación y otros males). De ellos, el $50 \%$ podrían haberse salvado con un tratamiento adecuado y oportuno" 62 . En este Programa contribuyeron el médico Carlos Avendaño, el médico jefe del Hospital Roberto del Río, el doctor Carlos Allendes, entre otros profesionales. Se eligió el Área Hospitalaria Norte porque "cuenta con una gran población expuesta al riesgo por las malas condiciones socio económicas y culturales en que viven; además, las diarreas son cuadros prevenibles y con acciones oportunas se disminuye el riesgo de agravamiento y muerte de los niños" ${ }^{\prime 63}$.

La complejidad de los problemas de salud infantil obligó a los gobiernos a expandir la supervisión médica de las madres y los niños mediante la instalación de policlínicas periféricas para descongestionar la demanda en los hospitales. Esto significaba difundir contenidos que fortalecieran el enfoque preventivo en materias de salud infantil e intentar reducir el número de potenciales pacientes que podrían colapsar los centros hospitalarios. Para el ministro de Salud, Ramón Valdivieso, uno de los principales déficit de la medicina era la falta de extensión de la medicina pediátrica en diversos barrios, donde "por cada tres fallecidos uno no tuvo la atención médica requerida" ${ }^{64}$. En ese marco, no bastaba que la ciencia estudiara las

\footnotetext{
${ }^{59}$ BN, El Siglo. Santiago, 11 de agosto de 1957.

${ }^{60}$ Guemes Riveros, Marta. 1945. Factores médicos y económicos-sociales que condicionan la mortalidad infantil en Chile, Santiago, Talleres Gráficos Simiente, p. 18; Cienfuegos, Eugenio. 1933. Estudios clínicos de pediatría, Santiago, Imprenta El Esfuerzo, p. 8.

61 Zárate, Soledad. 2018. "Señora, su hijo no va a morir: enfermeras y madres contra la mortalidad infantil, Chile, 1950-1980", en Cordero, Macarena, Rastros y gestos de las emociones, Santiago, Editorial Cuarto Propio, pp. 168 y ss.

62 BN, La Nación. Santiago, 24 de enero de 1967.

${ }^{63}$ BN, El Mercurio. Santiago, 18 de noviembre de 1962.

${ }^{64}$ BN, El Mercurio. Santiago, 12 de noviembre de 1964.
} 
enfermedades solo desde el ámbito médico, resultaba necesario el "estudio de aquellos factores médicos que tienen relación con los problemas sociales y de aquellas causas sociales que tienen influencia en el desarrollo de ciertas enfermedades" ${ }^{\prime 65}$.

La alta congestión de pacientes fue otro de los obstáculos en el funcionamiento de los consultorios establecidos en áreas periféricas. La razón de estas aglomeraciones fue la irregularidad en los horarios de atención de los médicos, lo que las autoridades explicaban porque "la mayoría de éstos trabaja en la mañana en hospitales, para después viajar a San Gregorio, La Granja u otras poblaciones. La falta de incentivos resta interés a los médicos jóvenes por ir a estos núcleos y prefieran sus trabajos en la zona céntrica de Santiago" ${ }^{66}$. La precaria situación del sistema hospitalario, la falta de personal especializado y de recursos materiales, generó junto a otras causales, un grave problema en los sectores de Lo Valledor, Unión Ferroviaria, José María Caro, las que prácticamente no tenían atención médica para 30 mil niños. Para estas zonas, solo se contaba con "una incompleta policlínica. Los médicos hacen lo imposible, pero no cuentan ni con los más elementales útiles de trabajo, y todo lo que hay lo han prestado ellos mismos. De 250 menores enfermos, deben rechazar todos los días a 70, los cuales no son atendidos en ninguna otra parte" ${ }^{67}$. Tenían una ambulancia y otras secciones que no se podían implementar por falta de personal competente ${ }^{68}$.

A partir de los datos entregados por los Anuarios Estadísticos de la República es posible establecer varios parámetros médicos y demográficos de la población de 1930 a 1973. Los volúmenes de esta publicación consignaban en la sección de estadística médica la cantidad de camas disponibles en cada uno de los hospitales. Fijado el número de las instalaciones en los establecimientos médicos infantiles (Roberto del Río, Manuel Arriarán y Luis Calvo Mackenna), cabe deducir que la cuantía de camas provinciales y nacionales tuvo un crecimiento constante en el período. Las tres primeras columnas expresan la cifra de camas hospitalarias, mientras que la cuarta y quinta muestran los totales de camas en la Provincia de Santiago y a nivel país, respectivamente. Ahora bien, la disposición de camas se triplicó, lo que conduce a suponer que la población mantuvo el mismo ritmo de crecimiento.

La misma tabla 2, incluye a continuación, una columna con las defunciones en la provincia. La relación entre el conjunto de fallecimientos de todas las edades y los menores de un año entrega información clave para evidenciar la reducción de la mortalidad infantil. En esa proporción, por ejemplo, para 1930, las defunciones totales fueron 24.680 y de ellas, las de menores de un año constituyeron 8.667 , lo que representaba un $35,12 \%$ de las muertes. En

\footnotetext{
65 Baeza Goñi, Arturo. 1950. Progresos de la pediatría y de la Medicina Social de la Infancia, Santiago, [s.n.], p. 15. ${ }^{66}$ BN, El Mercurio. Santiago, 18 de julio de 1964.

${ }^{67}$ BN, El Clarín. Santiago, 20 de junio de 1961.

68 ARNAD, Para mejorar los protocolos en los hospitales infantilles establecieron reglamentos para cursos de perfeccionamiento de las cuidadoras de niños. Para ver el reglamento completo del curso, véase: Santiago, 22 de mayo de 1939. vol. 224, s/f.
} 
cambio, para el año 1973, fallecieron 26.662 personas, donde los que murieron antes del año de vida fueron 4.423, lo que significaba 16,58 \% de los decesos. En suma, entre las cuatro décadas que abarca esta investigación, la mortandad se redujo desde 248.8 por cada 1000 nacidos vivos hasta llegar a la cifra de 48.2. De todo ello se puede deducir que el aumento de las instalaciones clínicas fue proporcional a la disminución de las muertes infantiles ${ }^{69}$.

Tabla 2. Distribución de camas para tratamientos médicos infantiles e indicadores demográficos Provincia de Santiago y Chile, 1930 - 1973

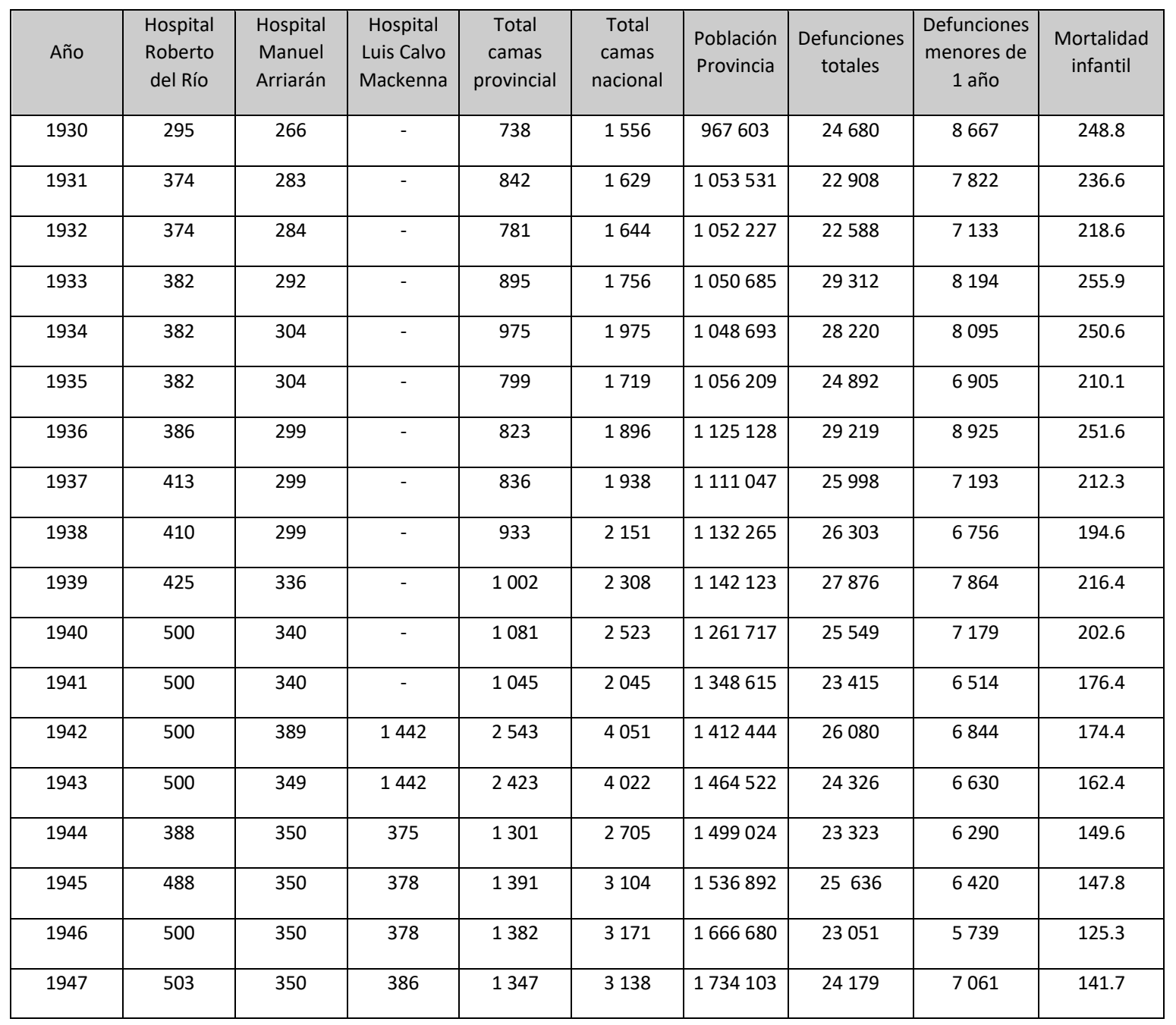

69 Kaempfer, Ana María y Medina, Ernesto. 1982. "La salud infantil en Chile durante la década del setenta”, Revista Chilena de Pediatría, vol. 53/5, Santiago, p. 471. 


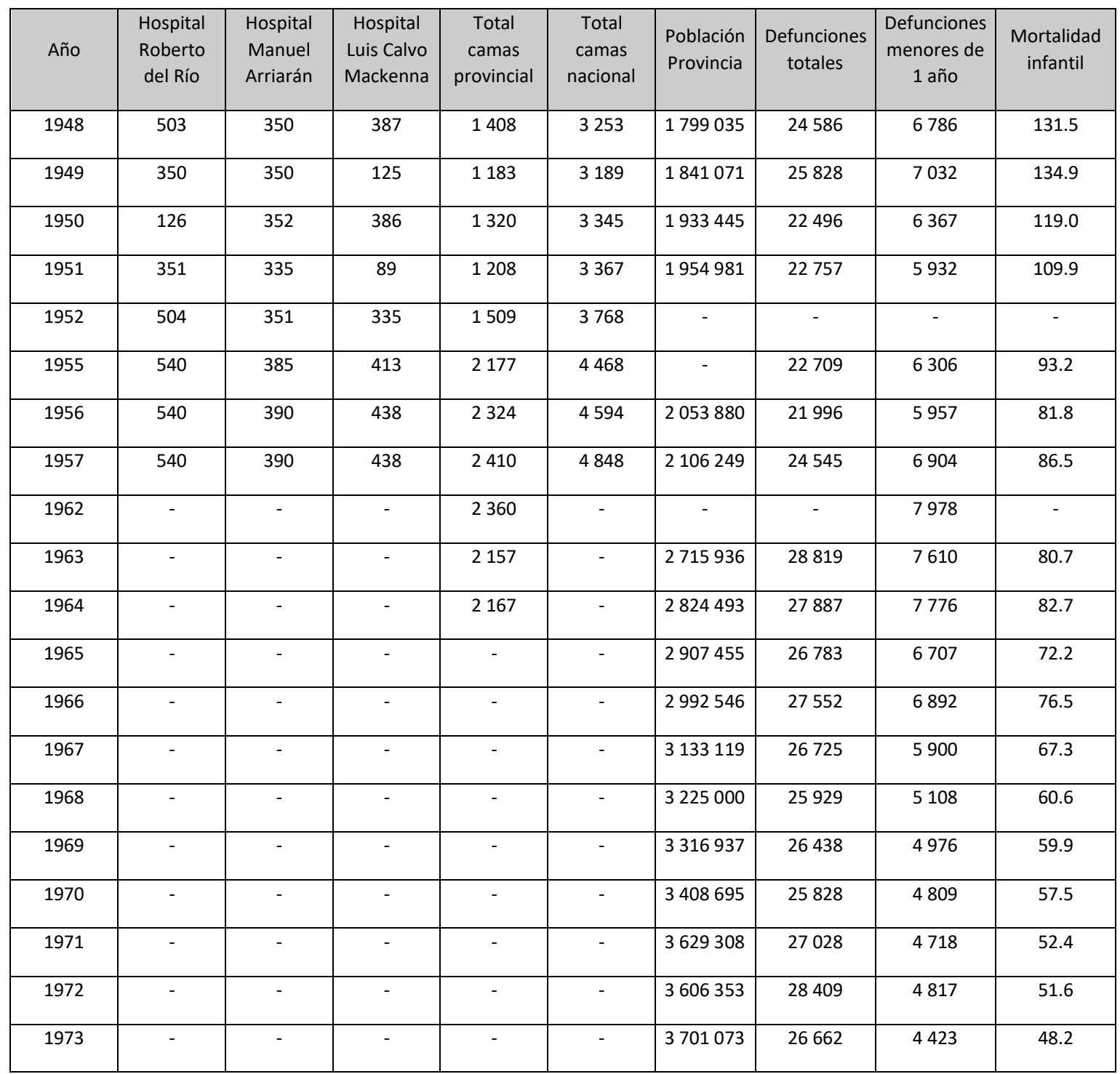

Fuente: Elaboración de los autores a partir del Anuario Estadístico de la República de Chile, 1930-1973.

\section{La atención hospitalaria de las enfermedades infantiles}

La salud infantil es un componente importante en el desarrollo de las sociedades y se encuentra en directa relación con la situación económica o la cantidad de recursos que tiene el país. De ahí surgen algunas preguntas, tales como ¿cuál es el monto de inversiones que el Estado dirige hacia las necesidades de salud? ¿El aumento de las inversiones en medicina se traduce en el mejoramiento substancial de los niveles de salud? ¿En qué ámbitos los mayores gastos rendirán 
beneficios máximos? Dichas interrogantes fueron ampliamente debatidas por los especialistas, estableciendo que "mayores gastos médicos inciden en los índices de mortalidad. No obstante se puede deducir que no hay una disminución manifiesta de la mortalidad como consecuencia de un mayor gasto médico. En otras palabras, el gasto médico, por si solo, no es un factor determinante de disminución de la mortalidad infantil" ${ }^{70}$. Así, la disminución de las muertes en la niñez estaría influida por las inversiones en salud, pero también era clave abordar las causales que permitieran mejorar las condiciones de vida de la población.

La fundación de la Sociedad Constructora de Establecimientos Hospitalarios (1944-1982) tuvo como objetivo el impulso de la construcción de recintos hospitalarios. En esta época, era vital la construcción de hospitales y el desarrollo de los planes de salubridad que atendieran a la defensa de la salud de las madres y los niños. Para apoyar estos proyectos, la Junta de Beneficencia acordó enajenar algunas de sus propiedades raíces y dirigir los capitales a los fondos de este organismo para aumentar la asistencia hospitalaria. En este período "el crecimiento de la población, la antigüedad de casi todos los edificios de hospitales, que ha obligado a abandonar partes completas, que ya se hacían inutilizables, y la extensión de los servicios que deben ampliarse de acuerdo con los nuevos conocimientos, han creado un problema hospitalario grave" ${ }^{71}$.

El impulso de Sociedad Constructora permitió la construcción de varios edificios hospitalarios, los que fueron equipados por las gestiones de la Corporación de Fomento de la Producción, la que mediante créditos logró adquirir una serie de equipos en Estados Unidos y Europa. El volumen de acción de esta entidad muestra que "en 1940 con solo 192 hospitales y 20.428 camas existentes, pasó en 1951 y por la decisiva contribución realizada de la Sociedad este número de establecimientos se eleva a más de 220, con una capacidad de 30.765 camas" $^{72}$. Todos estos progresos llevaron a la aplicación de nuevas tecnologías y métodos de tratamientos lo que tuvo como efecto una reducción en los períodos de hospitalización.

Los planes que fortalecían la red hospitalaria abarcaban la construcción de centros de salud en ciudades más pequeñas con el objetivo de realizar atención inmediata, de aprovechar los espacios en los que podía obtenerse la curación de la salud y distribuir los servicios profesionales de los médicos en el país. Cabe señalar que estos proyectos no solo consideraron la instalación de nuevos recintos, sino que organizaron su funcionamiento mediante la reducción del tiempo de permanencia de los enfermos, pues su convalecencia la harían en su propio domicilio, controlados por las asistencias ambulatorias. La extensión del trabajo

\footnotetext{
70 BN, El Mercurio. Santiago, 26 de septiembre de 1960.

71 BN, La Nación. Santiago, 9 de abril de 1949.

72 BN, La Nación. Santiago, 27 de octubre de 1952.
} 
obstétrico y pediátrico en la ciudad permitiría recabar información sobre la magnitud de los problemas y determinar los riesgos de enfermedades en la madre o el niño ${ }^{73}$.

La creación de la posta infantil en el Hospital Arriarán, representó un factor de transformación cultural en la atención médica de la salud infantil. Estas instalaciones aportaron prestaciones a los niños enfermos que necesitaban exámenes clínicos, particularmente en el diagnóstico de afecciones infantiles. Hasta ese momento, las urgencias de los infantes se trataban en la Asistencia Pública de Santiago en contacto con un flujo de pacientes con diversas enfermedades, lo que retardaba o complicaba una oportuna observación. Con esta nueva instalación, "los pequeños son atendidos por médicos especializados. Al mismo tiempo, se aprovechan los servicios auxiliares del hospital (laboratorio, rayos, transfusión, etc.)"74. Así, los infantes podían ser examinados de modo inmediato por enfermeras o pediatras y al ubicarse en un sector estratégico del hospital permitía la comunicación con múltiples secciones, por ejemplo las ambulancias podían ingresar directamente a la posta.

Una característica fundamental del sistema de salud infantil a mediados del siglo XX fue la extensión de los avances médicos hacia amplios segmentos de la población. Para el SNS representaba la piedra angular de toda estructura moderna de protección social ${ }^{75}$. La puesta en marcha de consultorios fue la base del reparto de alimentos mitigando el raquitismo de los infantes y aumentando la resistencia física frente a las desfavorables condiciones del medio ambiente ${ }^{76}$. Además, se logró el control de varias enfermedades por el empleo racional de antibióticos, vacunaciones y aplicación de medidas preventivas en estos servicios orientadas hacia la atención materno infantil, cuidando el correcto desarrollo del embarazo y el parto, controlando el mayor número posible de niños para reducir la elevada cifra de mortalidad en esta época de la vida, desarrollando campañas de educación sanitaria, de lucha contra enfermedades transmisibles ${ }^{77}$. Dicho enfoque estaba sustentado en varias acciones: las madres eran tratadas y examinadas periódicamente durante el embarazo, los partos se realizaban en establecimientos hospitalarios bajo observación médica y tanto la madre como el niño eran atendidos en el período siguiente al parto ${ }^{78}$.

\footnotetext{
${ }^{73}$ Szot, Jorge. 2002. "Reseña de la salud pública materno infantil chilena durante los últimos 40 años: 1960-2000", en Revista chilena de obstetricia y ginecología, vol. 67/2, Santiago, p. 131.

${ }_{74}$ BN, El Mercurio. Santiago, 28 de junio de 1943.

${ }^{75}$ BN, La Nación. Santiago, 31 de julio de 1958.

${ }^{76}$ Servicio Nacional de Salud. 1961. Ración recomendada durante el embarazo y la lactancia. Folleto destinado al personal del Servicio que participa en funciones educativas, Santiago, Servicio Nacional de Salud.

77 Rosselot, Jorge. 1965. Problemática de la protección materno-infantil en el Servicio Nacional de Salud de Chile, Santiago, Servicio Nacional de Salud, p. 10.

78 González, Maricela y Zárate, Soledad. 2019. "Trabajo, miserias y recompensas: Asistentes sociales, enfermeras y matronas en la construcción de la política sanitaria chilena a mediados del siglo XX", Salud Colectiva, vol. 15, Buenos Aires, pp.1-16; Zárate, Soledad y González, Maricela. 2018. "Matronas y la consolidación del parto hospitalario en Chile, 1950-1970", en Sánchez, Georgina y Laako, Hanna (editoras), Parterías de Latinoamérica. Diferentes territorios, mismas batallas, Chiapas-México, El Colegio de la Frontera Sur, pp. 34-69.
} 
Dada la extensión espacial de los barrios en la capital y el aumento de la población, las postas infantiles debían crecer rápidamente para atender a tan numeroso grupo de pacientes. En esos lugares, no solo era necesaria la construcción de nuevos hospitales y camas, ya que este crecimiento bajaba el rendimiento del personal especializado, al estar a su cargo un mayor número de enfermos ${ }^{79}$. Para el Director del Hospital Arriarán, "la solución sería que cada comuna contara con su hospital, porque así como cada una cuenta con sus escuelas, municipalidades y secciones de vigilancia, así debería tener sus servicios asistenciales" ${ }^{80}$.

Así se aplicaban los preceptos de la Organización Mundial de la Salud, los que ponían énfasis en el bienestar físico y no solo en la ausencia de enfermedad. En esa línea, el concepto de salubridad tenía como propósito el fomento, la protección y la reparación de la salud. Para el médico Arturo Baeza Goñi, "cuanto más efectiva sea la labor que se realice en las dos primeras etapas, repercutirá en reducir las proporciones del último. Es obvio, entonces, que una política eficaz debiera consistir en intensificar los organismos que pueden ejercer el máximo de beneficio mediante acciones preventivas para disminuir al mínimo las posibilidades de enfermar" ${ }^{\prime \prime}$.

\section{La distribución de especialistas pediátricos y las tecnologías hospitalarias}

Durante este período existía una insuficiencia de médicos en todas las especialidades. Según los datos en la tabla 3, la suma de los pediatras dispuestos en cada uno de los hospitales infantiles de la capital aumentaron paulatinamente, expresadas desde los 86 especialistas en 1930 hasta los 327 en 1957. Dicha variación marcó un avance importante con la apertura del hospital Luis Calvo Mackenna en 1942. No obstante, este crecimiento de los profesionales no guardaba relación con el aumento de la población, tomando en cuenta la cantidad de habitantes es posible calcular que el número de médicos pediatras hacia 1930 era inferior a 1 por cada 10.000 habitantes de la Provincia. En el caso de las matronas la situación era aún más dramática, las que se encontraban en razón de 0,6 por cada 10.000 habitantes y que, respecto de los médicos, no presentaron un alza tan considerable en esta época. De ese modo, a finales de la década de 1950 se experimentaba un aumento demográfico, especialmente infantil, debido al descenso de la mortalidad y el aumento de la esperanza de vida, lo que ocasionaba mayores demandas a los servicios hospitalarios y que muchos de ellos vieran superada su capacidad de brindar una correcta atención curativa.

En 1964, el Noveno Congreso Nacional de Pediatría se destinó a abordar cinco causales que incidían en la alta mortandad infantil. En este encuentro los médicos se ocuparon de revisar sus

\footnotetext{
${ }^{79}$ Hospital de Niños Roberto del Río, Departamento de Pediatría. 1973. Normas de atención médica intrahospitalaria, Santiago, Departamento de Pediatría, Área Norte, p. 8.

${ }^{80}$ BN, La Nación. Santiago, 8 de marzo de 1960.

${ }^{81}$ BN, El Mercurio. Santiago, 17 de junio de 1958.
} 
experiencias para fijar normas que tendieron a remediar estos peligros. El médico Adalberto Steeger, presidente de la Sociedad Chilena de Pediatría, señalaba que "faltan pediatras en Chile, como país joven, tiene una población infantil que equivale al $37 \%$ de la población total. En la misma proporción, las consultas médicas corresponden en un $50 \%$ a la pediatría. Frente a esta demanda poderosa, solo el $13.2 \%$ de los médicos se dedican a la pediatría. En circunstancias que debiéramos tener entre el 25 y $30 \%$ dedicados a la medicina infantil" ${ }^{82}$. La finalidad era establecer el número de consultas médicas infantiles promedio en la provincia, la calidad, la eficiencia y los establecimientos asistenciales para otorgarlas. La cifra de médicos y otros profesionales que serían indispensables para cubrir estas necesidades en el ámbito de salud.

La cantidad de médicos especialistas en los hospitales infantiles supone que hacia 1930 existían casi un médico especialista por cada 10.000 habitantes en la Provincia. Con la inauguración del hospital Luis Calvo Mackenna se superó la barrera y de ahí en adelante, la tendencia fue siempre mayor a uno, llegando en 1957 a la cantidad de 1,5 médicos por cada 10.000 habitantes. En el caso de las matronas, a pesar de mantener una inclinación al alza, la cantidad de ellas nunca sobrepasó una disposición superior a 1 por cada 10.000 habitantes. A partir de esta tabla es posible establecer que el crecimiento de especialistas médicos infantiles y matronas fue bastante distante respecto de las necesidades de la población. La situación se complejiza aún más al pensar en la distribución de los profesionales, lo más probable es que estaban disponibles solo en algunas zonas de la capital mientras que otros sectores carecían totalmente de estos servicios.

Tabla 3. Médicos distribuidos en hospitales infantiles y matronas en la Provincia de Santiago, 1930 - 1957

\begin{tabular}{|c|c|c|c|c|c|c|c|}
\hline Año & $\begin{array}{c}\text { Roberto del } \\
\text { Río }\end{array}$ & $\begin{array}{c}\text { Manuel } \\
\text { Arriarán }\end{array}$ & $\begin{array}{c}\text { Luis Calvo } \\
\text { Mackenna }\end{array}$ & Total médicos & $\begin{array}{c}\text { Número } \\
\text { médicos por } \\
10000 \text { hab. }\end{array}$ & $\begin{array}{c}\text { Total } \\
\text { matronas }\end{array}$ & $\begin{array}{c}\text { Número de } \\
\text { matronas por } \\
10 \text { 000 hab. }\end{array}$ \\
\hline 1930 & 40 & 46 & - & 86 & 0,888 & 58 & 0,599 \\
\hline 1931 & 40 & 43 & - & 83 & 0,787 & 58 & 0,550 \\
\hline 1932 & 41 & 43 & - & 84 & 0,798 & 59 & 0,560 \\
\hline 1933 & 41 & 43 & - & 84 & 0,799 & 54 & 0,513 \\
\hline 1934 & 44 & 46 & - & 90 & 0,858 & 53 & 0,505 \\
\hline 1935 & 44 & 47 & - & 91 & 0,861 & 65 & 0,615 \\
\hline 1936 & 44 & 49 & - & 93 & 0,826 & 77 & 0,684 \\
\hline
\end{tabular}

82 BN, La Nación. Santiago, 10 de noviembre de 1964. 


\begin{tabular}{|c|c|c|c|c|c|c|c|}
\hline 1937 & 39 & 47 & - & 86 & 0,774 & 77 & 0,693 \\
\hline 1938 & 39 & 49 & - & 88 & 0,777 & 81 & 0,715 \\
\hline 1939 & 46 & 53 & - & 99 & 0,866 & 92 & 0,805 \\
\hline 1940 & 50 & 54 & - & 104 & 0,824 & 87 & 0,689 \\
\hline 1941 & 54 & 55 & - & 109 & 0,808 & 87 & 0,645 \\
\hline 1942 & 54 & 65 & 38 & 157 & 1,111 & 82 & 0,580 \\
\hline 1943 & 55 & 66 & 45 & 166 & 1,113 & 86 & 0,587 \\
\hline 1944 & 54 & 67 & 45 & 166 & 1,107 & 95 & 0,633 \\
\hline 1945 & 58 & 67 & 48 & 173 & 1,125 & 89 & 0,579 \\
\hline 1946 & 67 & 70 & 44 & 181 & 1,085 & 96 & 0,575 \\
\hline 1947 & 86 & 71 & 50 & 207 & 1,193 & 95 & 0,547 \\
\hline 1948 & 86 & 71 & 50 & 207 & 1,150 & 107 & 0,594 \\
\hline 1949 & 89 & 74 & 44 & 207 & 1,124 & 108 & 0,586 \\
\hline 1950 & 89 & 64 & 57 & 210 & 1,086 & 115 & 0,594 \\
\hline 1951 & 89 & 74 & 55 & 218 & 1,115 & 112 & 0,572 \\
\hline 1952 & 89 & 74 & 55 & 218 & - & 120 & - \\
\hline 1955 & 127 & 96 & 100 & 323 & - & 192 & - \\
\hline 1956 & 127 & 96 & 104 & 327 & 1,592 & 196 & 0,954 \\
\hline 1957 & 127 & 96 & 104 & 327 & 1,552 & 204 & 0,968 \\
\hline
\end{tabular}

Fuente: Elaboración de los autores a partir del Anuario Estadístico de la República de Chile, 1930-1957.

A mediados del siglo XX, el funcionamiento de los recintos hospitalarios mostró la situación precaria en que se encontraban. Se expresaba en la falta de elementos indispensables para la curación de los enfermos, entre ellos la escasez de medicamentos, de camas e incluso los medios más básicos de alimentación. La prensa reproducía la compleja posición del Hospital Manuel Arriarán, donde escaseaba la ropa, no había fundas, frazadas, ni colchones para atender a una numerosa población. Por esta causa se debía rechazar a muchos enfermos. El doctor Carlos Izzo, médico jefe afirmaba que "mientras operaba a un pequeño, con su ayudante lo hacían sin guantes de goma. No tiene el hospital recursos para adquirirlos" ${ }^{183}$. En efecto, para los especialistas que trabajaban en la salud pública, las posibilidades de conseguir mejoras en los pacientes se veían dificultadas ante el déficit de material quirúrgico o útiles hospitalarios,

${ }^{83}$ BN, El Mercurio. Santiago, 5 de octubre de 1933. 
por lo que no es sorprendente que varios infantes no recibieran tratamiento médico o no les fuera posible obtener una curación durante su internación en los establecimientos.

La tabla 4 expresa la información sobre los movimientos hospitalarios infantiles entre 1930 y 1956. En estas casi tres décadas la cantidad de pacientes admitidos y asistidos crecieron más de tres veces, en cambio el número de fallecidos se mantuvo constante a pesar de la mayor demanda. En suma, entre 1930 y 1956, se establece el crecimiento del sistema hospitalario siguiendo las cifras de mortandad en la Provincia. Según los resultados de la tabla 2 para 1930 se produjeron 8.667 muertes de infantes menores de un año, de ellos 1.092 ocurrieron en recintos hospitalarios, lo que equivale a decir que el 12,59 \% sucedieron en hospitales; en el año 1956 se registraron 5.957 decesos de niños menores de un año, de ese total 1.483 acontecieron en establecimientos de salud, lo que significa que el $24,89 \%$ de los pequeños murieron en hospitales. La duplicación de la mortandad hospitalaria resulta de vital importancia para demostrar la inclusión de la estructura del sistema de salud en la población, o sea que las defunciones se producirían tras un tratamiento médico clínico y que iría en franco retroceso la ocurrencia en los hogares sin recibir asistencia médica.

Puede decirse que el proceso de incorporación de nuevas tecnologías y aparatos mejoró la calidad de la salud que se entregaba en los hospitales. En el caso de los niños con nacimiento prematuro se tomaban como parámetros el peso menor a 2,5 kilos y 43 centímetros para registrarlos en esta categoría. Los cuadros mostraban diferencias considerables, según la duración de la gestación. A nivel médico:

"Las reglas generales para asistir a los niños prematuros son: la protección contra el frío y el calor; la alimentación adecuada y la profilaxis contra las infecciones. Como el metabolismo es bajo, la deficiencia debe suplirse por la aplicación de calor. Esto se consigue mediante la incubadora. La cama de Hess se usa frecuentemente. Una incubadora práctica puede hacerse con una cesta cerrada, o una caja atemperada con botellas de agua caliente o con lámparas de luz eléctrica" ${ }^{84}$.

\footnotetext{
${ }^{84}$ Zahorsky, John and Zahorsky, Theodore Saunders. 1940. Sinopsis de pediatría. Primera edición en español, México, Unión Tipográfica Editorial Hispano-Americana, p. 540.
} 
Tabla 4. Indicadores Hospitales Roberto del Río, Manuel Arriarán y Luis Calvo Mackenna $1930-1956$

\begin{tabular}{|c|c|c|c|}
\hline Año & Admitidos & Asistidos & Fallecidos \\
\hline 1930 & 5619 & 6082 & 1092 \\
\hline 1931 & 5433 & 5969 & 1054 \\
\hline 1932 & 5558 & 6030 & 1050 \\
\hline 1933 & 5821 & 6265 & 1073 \\
\hline 1934 & 6435 & 6996 & 1015 \\
\hline 1935 & 6469 & 6999 & 882 \\
\hline 1936 & 6710 & 7253 & 1004 \\
\hline 1937 & 6309 & 6848 & 733 \\
\hline 1938 & 7096 & 7598 & 760 \\
\hline 1939 & 7921 & 8510 & 1044 \\
\hline 1940 & 7666 & 8252 & 813 \\
\hline 1941 & 7952 & 8587 & 621 \\
\hline 1942 & 10729 & 11460 & 1028 \\
\hline 1943 & 9695 & 11251 & 883 \\
\hline 1944 & 10511 & 11441 & 821 \\
\hline 1945 & 11918 & 12863 & 888 \\
\hline 1946 & 8768 & 6899 & 902 \\
\hline 1947 & 13073 & 14039 & 1102 \\
\hline 1948 & 12584 & 13592 & 1049 \\
\hline 1949 & 12678 & 13754 & 792 \\
\hline 1950 & 12857 & 13906 & 744 \\
\hline 1951 & 13526 & 14356 & 894 \\
\hline 1955 & 19909 & 21106 & 1298 \\
\hline 1956 & 21004 & 21935 & 1483 \\
\hline
\end{tabular}

Fuente: Elaboración de los autores a partir del Anuario Estadístico de la República de Chile, 1930-1956.

Estos progresos técnicos mejoraron el rendimiento de la medicina y una especialización que llevaron a disminuir la morbimortalidad infantil. En la primera mitad del siglo XX, el desarrollo de aparatos como la incubadora y el pulmón artificial permitió salvar a muchas criaturas que sin estas técnicas habrían fallecido por inmadurez en el nacimiento o asfixia. De ese modo, se 
presentaba una estrecha relación entre el peso de nacimiento y mortalidad ${ }^{85}$. Las cifras entregadas por el médico Arturo Baeza Goñi, "cada año nacen en Chile 250 mil niños, de los cuales 25.000 nacen prematuros, con desarrollo insuficiente y escasas probabilidades de vida. Por cada 10.000 nacidos vivos, mueren 30 madres. Frente a eso, el Servicio Nacional de Salud, desde 1952 a 1957, ha aumentado el número de atenciones prenatales de 280.000 a 440.000 y el de consultas de lactantes de 1.100 .000 a $2.200 .000^{\prime \prime 6}$.

Esta modificación estructural llevada a cabo por el SNS tuvo ciertos efectos en la mortalidad infantil, pues el descenso de las tasas se explicaba por la mayor eficacia de las prestaciones médicas, dada "la mayor preocupación por hacer realidad la entrega de la cuota de leche, que a todo recién nacido la ley le asegura" ${ }^{87}$. Sumada a la distribución de leche para mujeres embarazadas y nodrizas. Paralelamente, se puso en práctica la expansión de la asistencia médica de salud infantil para reforzar los barrios que se encontraban más carentes de este tipo de atención. En esa línea, el personal especializado del servicio realizó encuestas a varias madres y sus resultados indicaban que "existe ignorancia en cuanto a los más elementales principios de higiene en la preparación de los alimentos para niños. Un elevado porcentaje de mujeres no se lava las manos al salir del baño, no protege la leche de las moscas, no tiene las uñas limpias y guarda las mamaderas con restos de la última papa" ${ }^{88}$. Así, estas enfermedades podían llevar muchas veces a los menores al hospital, pero al retornar al medio ambiente con escasas normas de limpieza probablemente volverían a tener afecciones gastrointestinales ${ }^{89}$. Tal como expresaba, en una sesión del Senado, el médico Salvador Allende, "a mayor pobreza más enfermedad, y a mayor enfermedad más pobreza" ${ }^{90}$.

El SNS no solo implementó medidas cuyo objetivo era el aumento del número de horas médicas destinadas a la atención infantil sino que llevó a cabo una serie de medidas de prevención relacionadas con las siguientes finalidades "higiene ambiental, eliminación de excremento, basuras y moscas; control de alimentos, manipulación en el hogar y en los sitios de producción; fomento de la salud, control de niño sano y alimentación suplementaria; recuperación de la salud, atención médica oportuna y precoz" ${ }^{91}$. Todas estas acciones llevaron a descender los estragos que provocaban las diarreas infantiles y evitar los efectos más graves de esta enfermedad. Así, debido a los avances en las condiciones de higiene y el freno a los

\footnotetext{
85 Ipinza, Iván. 1952. Peso del recién nacido y sus relaciones con la gestación y el parto, Santiago, Tesis para optar al título de Licenciado en Medicina; Ortega, Raúl. 1944. El prematuro en las clínicas y en la literatura médica norteamericana, Santiago, Imprenta de Carabineros, p. 8.

${ }^{86} \mathrm{BN}$, El Mercurio. Santiago, 2 de agosto de 1958.

${ }^{87}$ BN, La Nación. Santiago, 9 de abril de 1966.

88 BN, La Nación. Santiago, 24 de enero de 1967.

89 Sievers, Hugo. 1934. El problema de la leche higiénica, Santiago, Prensas de la Universidad de Chile, p. 15.

90 BN, El Clarín. Santiago, 9 de noviembre de 1962.

${ }^{91}$ BN, La Nación. Santiago, 10 de diciembre de 1971.
} 
brotes de enfermedades infecciosas, ocasionando "la disminución de la mortalidad infantil. Siendo una de las victorias más hermosas sobre la muerte" ${ }^{92}$.

En este período el gobierno de Chile, la Organización Mundial de la Salud y la UNICEF firmaron un convenio destinado a la ejecución de un plan de operaciones para la vigilancia de nacidos antes del término de la gestación ${ }^{93}$. Las finalidades del proyecto eran "reducir las cifras de mortalidad de niños prematuros, mejorar su atención, establecer un centro de demostración y adiestramiento de profesionales y auxiliares, que funcionará en el hospital Calvo Mackenna. La UNICEF, de acuerdo con este compromiso, proporcionará 10 incubadoras, estufas eléctricas, refrigeradores, máquinas lavadoras y esterilizadoras"94. Este desarrollo de normas y procedimientos abarcó el cuidado de los prematuros en el plan de servicios de salud pública. Años más tarde, un nuevo convenio entre el gobierno de Chile y la UNICEF contempló "un aporte a nuestro país de tres millones 198 mil dólares con el objetivo principal de extender el programa de atención integral desde el punto de vista médico social, tanto en el parto como en los cuidados posteriores de la madre y del recién nacido, contribuyendo de este modo al bienestar familiar" ${ }^{\prime 5}$.

Con el trabajo de los hospitales también se obtuvieron efectos positivos en los cuidados médicos a los nacidos prematuros. En la práctica, "sale del Hospital Calvo Mackenna, una camioneta llevando transportadoras donde conducirán al niño prematuro hasta el Centro especial. En el vehículo, junto con esta maleta incubadora, llevan una bolsa con oxígeno para solucionar a tiempo las crisis respiratorias que afectan constantemente a estos pequeños" ${ }^{96}$. En estas situaciones el tiempo resultaba esencial, las dificultades respiratorias podían ocasionar el fallecimiento y, en algunos casos dañaría las células cerebrales por falta de oxígeno generando secuelas futuras. Es posible asegurar que las incubadoras jugaban un rol clave al mantener la humedad y los niveles de oxígeno para evitar que en el recién nacido se acumulen mucosidades que podrían llegar a los pulmones. Una vez que transcurre el período más complejo, el incubado comienza a subir de peso y su delicado organismo es capaz de regular mejor su temperatura, dando paso a una etapa en que es oportuno alimentarlo de modo autónomo.

\footnotetext{
92 Nicolai, Jorge. 1934. Mortalidad infantil y natalidad, Santiago, prensas de la Universidad de Chile, p. 12.

${ }^{93}$ La UNICEF también contribuyó con programas materno infantiles para la distribución de leche y la construcción de una Planta de Leche en San Fernando. Santiago, 19 de enero de 1952. ARNAD, vol. 1 205, s/f.

${ }^{94} \mathrm{BN}$, El Mercurio. Santiago, 2 de mayo de 1955.

${ }_{95}$ BN, La Nación. Santiago, 9 de junio de 1972.

${ }^{96}$ BN, La Nación. Santiago, 13 de octubre de 1964.
} 


\section{Conclusiones}

La mortalidad en la infancia como fenómeno de características biológicas y sociales se encontraba influido por múltiples factores médicos, económicos, sociales, jurídicos, culturales y ambientales. En este artículo se ha discutido acerca del rol que tuvo el sistema hospitalario en la reducción de estas cifras, específicamente el control del embarazo, el parto y la vigilancia médica en los primeros años de vida. Estos recintos se transformaron en espacios dedicados a la investigación y desde los cuales eran difundidos los progresos médicos. Estas dinámicas estuvieron apoyadas por la Sociedad de Pediatría, el desarrollo de congresos nacionales e internacionales, donde los especialistas intercambian experiencias y conocimientos sobre los cuidados de la salud en los infantes.

Las enormes cifras de mortalidad en las primeras etapas de la vida era un índice revelador del problema del abandono en la infancia, tanto por las estadísticas como por sus expresiones más preocupantes como la vagancia y la mendicidad. En ese marco, el surgimiento de la medicina social estudió y propuso soluciones a problemáticas como la lucha anti-tuberculosa, higiene del trabajo y la industria. En cuanto a la asistencia infantil, se reconocían causas directas o médicas, o sea las enfermedades que ocasionaban la muerte o dañaban la salud en esa edad, y también los factores indirectos o sociales, como la excesiva natalidad, la mala habitación y la pobreza económica en amplios sectores de la población. Así, se establecieron medios de defensa de la salud que consistieron en la organización y distribución de los organismos directos de asistencia desde la atención prenatal hasta el escolar, a través de servicios de consultorios, maternidades, hospitales, maternidades domiciliarias o centros dedicados al cuidado de niños.

La profundización de políticas de prevención de enfermedades demandó una gran inversión de recursos públicos y centrar el enfoque en los cuidados de la infancia. Este problema de salud pública era bastante amplio y complejo, pues requería observar la vida desde la gestación, mediante la atención adecuada de la futura madre. A continuación, resultaba necesario el examen del lactante, el infante y la madre. Durante estas etapas se tratan no solo las afecciones, sino también sus estados previos, junto con procurar una correcta nutrición, la higienización de la vivienda y el entorno en que habitaban los infantes.

Con la consolidación del SNS se inició una profunda campaña de protección materno infantil en los segmentos más desamparados de la sociedad, a fin de frenar las altas tasas de mortandad en la infancia. Una de las situaciones más críticas se produjo con los nacimientos prematuros, los que en su mayoría fallecían en la primera semana de vida. A finales de la década de 1960, la Organización Mundial de la Salud recomendaba dos caminos para encarar esta problemática de salud pública, en primer lugar, intensificar las medidas para la reducción de la pobreza y la subalimentación, y, en segundo término, la extensión de centros asistenciales que permitieran 
el contacto entre médicos y la comunidad sobre todo durante la etapa del embarazo, el parto y los primeros meses tras el nacimiento.

\section{Fuentes y bibliografía}

\section{Fuentes}

Archivo Nacional de la Administración (ARNAD)

Fondos de Ministerios y Servicios Públicos, Ministerio de Salud

Vol. 17, 110, 224, 387, 455, 470, 1 195, 1205.

Salón Camilo Henríquez, Biblioteca Nacional (BN)

El Clarín, Santiago $(1961,1962,1967)$

El Diario Ilustrado, Santiago (1937)

El Mercurio, Santiago (1930, 1933, 1938, 1941, 1943, 1945, 1953, 1955, 1958, 1960, 1962, 1963, 1964)

El Siglo, Santiago $(1947,1957)$

La Nación, Santiago (1933, 1936, 1937, 1940, 1945, 1949, 1952, 1958, 1960, 1964, 1966, 1967, 1971, 1972)

La Opinión, Santiago (1943)

\section{Bibliografía}

Armus, Diego. 2007. La ciudad impura. Salud, tuberculosis y cultura en Buenos Aires, 1870-1950, Buenos Aires, Edhasa.

Baeza Goñi, Arturo. 1950. Progresos de la pediatría y de la Medicina Social de la Infancia, Santiago, Imprenta El Esfuerzo.

Behm Rosas, Hugo. 1962. Mortalidad infantil y nivel de vida, Santiago, Ediciones de la Universidad de Chile.

Benedetti, Laura. 2019. "El hambre, la miseria y la escuela: el surgimiento de las organizaciones de auxilio a la infancia escolar pobre de Concepción (1917-1929)", en Revista Historia Universidad de Concepción, № 26/2, Concepción, pp. 33-52.

Bernabeu, Josep, Fariñas, Diego, Sanz, Alberto y Robles, Elena. 2003. "El análisis histórico de la mortalidad por causas. Problemas y soluciones”, en Revista de Demografía Histórica, vol. XXI/1, Barcelona, pp. 167-193.

Birn, Anne-Emanuelle. 2007. "Child health in Latin America: historiographic perspectives and challenges", em História, Ciências, Saúde-Manguinhos, vol. 14/3, Río de Janeiro, pp. 677-708.

Birn, Anne-Emanuelle. 2008. "Doctors on record: Uruguay's infant mortality stagnation and its remedies, 1895-1945", in Bulletin of the History of Medicine, № 82, Maryland, pp. 311-354.

Bustamante, Werner. 1939. Contribución al estudio del recién nacido y del lactante chileno (Santiago), Universidad de Chile, Tesis para optar al título de médico cirujano.

Carbonetti, Adrián y Peranovich, Andrés. 2007. "La mortalidad infantil en la ciudad de Córdoba entre principios y mediados del siglo XX", en Carbonetti, Adrián (comp.), Historias de enfermedad en Córdoba desde la colonia hasta el siglo XX, Córdoba, Programa de Salud y Enfermedad, Centro de Estudios Avanzados, U.N.C., pp. 75-93. 
Cavieres, Eduardo. 1999. "Salud Pública. Estrategias, políticas fiscales y cambio cultural en la reducción de la mortalidad. Valparaíso, 1920-1960", en Cuadernos de Historia, № 19, Santiago, pp. 195-224.

Cavieres, Eduardo. 2001. "Ser infante en el pasado. Triunfo de la vida o persistencia de estructuras sociales. La mortalidad infantil en Valparaíso, 1880-1950", en Revista de historia social y de las mentalidades, № 5, Santiago, pp. 31-58.

Chávez, Pablo. 2020. "La mortalidad infantil: entre la alimentación y las enfermedades gastrointestinales en Santiago (1880-1920)", en Cuadernos de Historia, № 52, Santiago, pp. 69-101.

Chávez, Pablo y Brangier, Víctor. 2020. "Esa diaria gotera de la muerte: Mortalidad infantil y los albores de la medicina pediátrica en la provincia de Santiago, Chile, 1870-1913", en Salud Colectiva, vol. 16, Buenos Aires, pp. 1-16.

Chávez, Pablo y Soto, José. 2019. "Historia de la mortalidad infantil en la Casa de Huérfanos de Santiago (1898-1923), en Anuario de Estudios Americanos, vol. 76/2, Sevilla, pp. 645-677.

Chávez, Pablo y Soto, José. 2019. "La primera estadística sanitaria infantil de la provincia de Santiago de Chile (1860-1929)", en Dynamis, vol. 39/2, Barcelona, pp. 149-174.

Cienfuegos, Eugenio. 1933. Estudios clínicos de pediatría, Santiago, Imprenta El Esfuerzo.

Di Liscia, María Silvia. 2002. "Hijos sanos y legítimos: sobre matrimonio y asistencia social en Argentina (1935-1948)", em História, Ciências, Saúde-Manguinhos, vol. 9, Río de Janeiro, pp. 209-232.

Ehrick, Christine. 2001. "Mothers and the Colossal Machine: Feminism, Social Assistance and the State in Uruguay, 1910-1932", in The Americas, vol. 58/1, pp. 121-139.

Figueroa, Ernesto. 1951. El consultorio externo del Hospital de niños y su misión médico-social, Santiago, [s.n.].

Gallo, Óscar y Márquez, Jorge. 2011. "La mortalidad infantil y la medicalización de la infancia. El caso de Titiribí, Antioquía, 1910-1950”, en Revista Historia y Sociedad, № 20, Medellín, pp. 57-89.

González, Maricela y Zárate, Soledad. 2019. "Trabajo, miserias y recompensas: Asistentes sociales, enfermeras y matronas en la construcción de la política sanitaria chilena a mediados del siglo XX", en Salud Colectiva, vol. 15, Buenos Aires, pp.1-16.

Guemes Riveros, Marta. 1945. Factores médicos y económicos-sociales que condicionan la mortalidad infantil en Chile, Santiago, Talleres Gráficos Simiente.

Illanes, María Angélica. 1993. En el nombre del pueblo, del estado y de la ciencia: historia social de la salud pública, Chile, 1880-1973: hacia una historia social del Siglo XX, Santiago, Colectivo de Atención Primaria.

Illanes, María Angélica. 1999. “Maternalismo popular e hibridación cultural en Chile 1900-1920”, en Nomadías, № 1, Santiago, pp.185-211.

Ipinza, Iván. 1952. Peso del recién nacido y sus relaciones con la gestación y el parto, Universidad de Chile, Santiago, Tesis para optar al título de Licenciado en Medicina.

Kaempfer, Ana María y Medina, Ernesto. 1982. "La salud infantil en Chile durante la década del setenta", en Revista Chilena de Pediatría, vol. 53/5, Santiago, pp. 468-480.

Kiple, Kenneth. 1989. "The Nutritional Link with Slave Infant and Child Mortality in Brazil", in The Hispanic American Historical Review, vol. 69/4, pp. 677-690.

Lagos, Rubén. 1952. El control prenatal y el recién nacido, Universidad de Chile, Santiago, Tesis para optar al grado de Licenciado en Medicina.

Llanos, Claudio y Lanfranco, María Fernanda. 2017. "La discusión política sobre la mortalidad infantil en Chile durante la década de 1930. Elementos para una aproximación histórico política", en Anuario de Estudios Americanos, vol. 74/2, Sevilla, pp. 675-703.

Luna Freire, María. 2008. "Ser mãe é uma ciência': mulheres, médicos e a construção da maternidade científica na década de 1920", em História, Ciências, Saúde-Manguinhos, vol. 15, Río de Janeiro, pp. 153-171. 
Luna Freire, María. 2010. "A construção da "maternidade científica" no Brasil republicano: Mulheres, mães e médicos: discurso maternalista no Brasil", en Estudos Feministas, vol. 18/3, Florianópolis, pp. 941-964.

Meneghello, Julio. 1960. Lecciones de pediatría. Tomo II: patología y clínica del prematuro y lactante, Santiago, Editorial Universitaria.

Meneghello, Julio, Rosselot, Jorge y Manterola, Alejandro. 1961. Lecciones de pediatría clínica y social. Puericultura individual y colectiva, Santiago, Editorial Universitaria.

Milanich, Nara. 2009. Children of fate. Childhood, class, and the State in Chile, 1850-1930, Durham, Duke University Press.

Milanich, Nara. 2011. "Women, children, and domestic labor in nineteenth-century Chile", in Hispanic American Historical Review, vol. 91/1, Durham, pp. 29-62.

Nunes, Eduardo. 2012. "The lives of children and their public intimacy: social work as a new factor in attending to the needs of children in Latin America, 1928-1948", em História, Ciências, SaúdeManguinhos, vol. 19/2, Río de Janeiro, pp. 1-23.

Nicolai, Jorge. 1934. Mortalidad infantil y natalidad, Santiago, prensas de la Universidad de Chile.

Ortega, Raúl. 1944. El prematuro en las clínicas y en la literatura médica norteamericana, Santiago, Imprenta de Carabineros.

Osta, María Laura. 2016. "Niños y niñas, expósitos y huérfanos en Montevideo del siglo XIX”, en Revista de la Facultad de Derecho, № 41, Montevideo, pp. 155-189.

Palma, Patricia. 2009. "Mortalidad infantil en Chile. La situación médica social en la Casa de Expósitos de Santiago 1889-1899", en Anales de historia de la medicina, vol. 19/2, Santiago, pp. 127-137.

Ponce de León, Macarena. 2007. La Reforma de la Caridad Ilustrada: del Socorro Intramuros al Socorro Extramuros. Prácticas de Caridad en Santiago, 1830-1880, Pontificia Universidad Católica de Chile, Tesis para optar al Grado de Doctor en Historia.

Rodríguez, Esteban. 1998. "La construcción de la salud infantil. Ciencia, medicina y educación en la transición sanitaria en España”, en Cuadernos de Historia Contemporánea, № 18, Madrid, pp. 19-52.

Rodríguez, Esteban. 2003. "La salud infantil, asunto ejemplar en la historiografía contemporánea", en Revista Dynamis, № 23, Barcelona, pp. 27-36.

Rojas, Jorge. 1996. Los niños cristaleros: trabajo infantil de la industria. Chile, 1880-1950, Santiago, Dirección de Bibliotecas, Archivos y Museos.

Rojas, Jorge. 1999. "Trabajo infantil en la minería: apuntes históricos", en Historia, vol. 32, Santiago, pp. 367-441.

Rojas, Jorge. 2001. "Los niños y su historia: un acercamiento conceptual y teórico desde la historiografía", en Pensamiento Crítico. Revista electrónica de historia, № 1, Santiago, pp. 1-39.

Rojas, Jorge. 2010. Historia de la infancia en el Chile republicano: 1810-2010, Santiago, Junta Nacional de Jardines Infantiles, 2010.

Rosselot, Jorge. 1965. Problemática de la protección materno-infantil en el Servicio Nacional de Salud de Chile, Santiago, Servicio Nacional de Salud.

Ruff, Claudio, Matheu, Alexis, Ruiz, Marcelo, Benites, Luis y Coral, Gabriel. 2018. "Fundamentos de la gestión del Capital Humano en entidades hospitalarias", en Revista Cubana de Investigaciones Biomédicas, vol. 37/2, Ciudad de la Habana, pp. 39-45.

Salazar, Gabriel. 2007. Ser niño "huacho" en la historia de Chile (siglo XIX), Santiago, LOM Ediciones.

Salazar, Gabriel y Pinto, J. 2002. Historia contemporánea de Chile V. Niñez y juventud, construcción cultural de actores emergentes, Santiago, LOM Ediciones.

Scroggie, Arturo. 1958. Tratado de pediatría: enfermedades infecciosas, Santiago, Editorial Andrés Bello, Editorial Universitaria. 
Servicio Nacional de Salud. 1961. Ración recomendada durante el embarazo y la lactancia. Folleto destinado al personal del Servicio que participa en funciones educativas, Santiago, Servicio Nacional de Salud.

Sievers, Hugo. 1934. El problema de la leche higiénica, Santiago, Prensas de la Universidad de Chile.

Szot, Jorge. 2002. "Reseña de la salud pública materno infantil chilena durante los últimos 40 años: 19602000", en Revista chilena de obstetricia y ginecología, vol. 67/2, Santiago, pp. 129-135.

Zahorsky, John and Zahorsky, Theodore Saunders. 1940. Sinopsis de pediatría. Primera edición en español, México, Unión Tipográfica Editorial Hispano-Americana.

Zárate, Soledad. 2005. "Parto, crianza y pobreza en Chile", en Sagredo, Rafael y Gazmuri, Cristián (dir.) Historia de la vida privada en Chile. Tomo III: el Chile contemporáneo de 1925 a nuestros días, Santiago, Aguilar Chilena Ediciones - Taurus, pp. 13-47.

Zárate, Soledad. 2007. "De partera a matrona. Hacia la asistencia profesional del parto en Chile en el siglo XIX", en Calidad en la educación, № 27, Santiago, pp. 284-297.

Zárate, Soledad. 2008. Dar a luz en Chile, siglo XIX. De la "ciencia de la hembra" a la ciencia obstétrica, Santiago, Ediciones Universidad Alberto Hurtado.

Zárate, Soledad. 2010. "El licor de la vida. Lactancia y alimentación materno-infantil en Chile, 1900-1950", en Sciolla, Carolina (comp.), Historia y cultura de la alimentación en Chile, Santiago, Catalonia, pp. 235-261.

Zárate, Soledad. 2018. "Señora, su hijo no va a morir: enfermeras y madres contra la mortalidad infantil, Chile, 1950-1980", en Cordero, Macarena, Rastros y gestos de las emociones, Santiago, Editorial Cuarto Propio, pp. 165-198.

Zárate, Soledad. y González, Maricela. 2018. “Matronas y la consolidación del parto hospitalario en Chile, 1950-1970", en Sánchez, Georgina y Laako, Hanna (eds.), Parterías de Latinoamérica. Diferentes territorios, mismas batallas, Chiapas-México, El Colegio de la Frontera Sur, pp. 34-69. 